\title{
Experience Alters the Spatial Tuning of Auditory Units in the Optic Tectum during A Sensitive Period in the Barn Owl ${ }^{1}$
}

\author{
ERIC I. KNUDSEN \\ Department of Neurobiology, Stanford University School of Medicine, Stanford, California 94305
}

\begin{abstract}
The auditory spatial tuning of bimodal (auditory-visual) units in the optic tectum of the barn owl was altered by raising animals with one ear occluded. Changes in spatial tuning were assessed by comparing the location of a unit's auditory best area with that of its visual receptive field. As shown previously, auditory best areas are aligned with visual receptive fields in the tecta of normal birds (Knudsen, E. I. (1982) J. Neurosci. 2: 1177-1194). It was demonstrated in this study that, when birds were raised with one ear occluded, best areas and visual receptive fields were aligned only as long as the earplug was in place. When the earplug was removed, best areas and visual receptive fields became misaligned, indicating that a change in auditory spatial tuning had taken place during the period of occlusion. However, in a bird that received an earplug as an adult, no such alterations in auditory spatial tuning were observed; even after 1 year of monaural occlusion, auditory best areas and visual receptive fields were misaligned so long as the earplug was in place, and were aligned when the earplug was removed. These results suggest that exposure to abnormal localization cues modifies the auditory spatial tuning of tectal units only during a restricted, sensitive period early in development.

After the earplug was removed from a juvenile bird that had been raised with an occluded ear, the initial misalignment between auditory best areas and visual receptive fields decreased gradually over a period of weeks. In contrast, when earplugs were removed from two adult birds that had been raised with monaural occlusions, auditory-visual misalignments persisted for as long as measurements were made, which was up to 1 year after earplug removal. These data indicate that auditory cues become permanently associated with locations in visual space during a critical period which draws to a close at about the age when the animal reaches adulthood.
\end{abstract}

Horseradish peroxidase was injected into two optic tecta (in a single animal) that contained units with permanently altered auditory spatial tuning. The positions of retrogradely labeled cells in the external nucleus of the inferior colliculus

Received March 22, 1985; Revised May 10, 1985;

Accepted May 13, 1983

\footnotetext{
'I thank Phyllis Knudsen for technical assistance and Drs. Sascha duLac, Steven Esterly, John Middlebrooks, Masakazu Konishi, Carla Shatz, Andrew Moiseff, Terry Takahashi, and Edward Sullivan for reviewing the manuscript. I also thank Brenda Robertson for typing the manuscript. This work was supported by grants from the March of Dimes (Grant 1-863), the Sloan Foundation, the National Institutes of Health (Grant RO1 NS 16099-06), and a Neuroscience Development Award from the McKnight Foundation.
}

(ICX) were the same as those observed in control birds (Knudsen, E. I., and P. F. Knudsen (1983) J. Comp. Neurol. 218: 187-196). Thus, the changes in spatial tuning were not due to a shift in the topographic projection from the ICX to the optic tectum. Based on these data and other arguments, it is concluded that the experience-dependent alterations in auditory spatial tuning must take place at an earlier stage in the auditory pathway, at or before the level of the ICX.

Early monaural occlusion altered sound localization behavior in a way that was similar to the alterations it induced in the spatial tuning of tectal units assessed in the same individuals (two animals). This suggests that similar neuronal modifications underlie both the physiological and behavioral effects of early monaural occlusion.

In the developing nervous system, sensory experience can play an important role in determining the response properties of central neurons. Previous work has shown that sensory experience is required for the maintenance or expression of unit properties that are innately determined (Hubel and Wiesel, 1970; Olson and Pettigrew, 1974; Daw and Wyatt, 1976; Stryker et al., 1978). The current study describes adaptive changes in the spatial tuning of central auditory units that are guided by early sensory experience. In this case, sensory experience modifies unit properties in such a way as to customize them to the sensory environment of the individual.

The auditory systern derives spatial informalion from a variely of acoustic cues. The most important cues are the frequency-specific differences in the timing and intensity of sound at the two ears that the auditory system evaluates and associates with appropriate locations in space. Because the correspondence between auditory cues and sound source locations depends upon the size and shape of the head and ears, the neural circuitry that associates these cues with locations must be calibrated for the physical acoustic properties of the species, and probably of the individual.

In barn owls, a neurophysiological assay of whether auditory cues are associated with correct locations is provided by the spatial tuning of auditory-visual bimodal units in the optic tectum (the homologue of the mammalian superior colliculus). These bimodal units are highly selective for sound source location, responding strongly to sounds only when the source is located in a restricted "best area," and their visual receptive fields align closely with their best areas (Knudsen, 1982). This alignment of auditory and visual spatial sensitivity represents an association of auditory cues with appropriate locations in visual space: each tectal unit is tuned to those auditory cues which correspond to the same location as its visual receptive field.

In experiments reported previously, the correspondence between auditory cues and visual locations was altered chronically in baby owls by occluding one ear (Leibovic and Knudsen, 1982; Knudsen, 1983a). Despite the disruption of auditory localization cues, tectal 
units recorded 5 to 10 months later with the earplug in place exhibited auditory best areas which aligned fairly well with visual receptive fields, suggesting that the auditory tuning of these units had changed. Accordingly, removal of the earplug caused auditory best areas to shift away from the side of the previously plugged ear and out of alignment with visual receptive fields. This misalignment persisted in adult birds for at least 5 months. These data indicate that the associations of auditory cues with locations in visual space can be changed by sensory experience during an early period in development. However, they leave a number of fundamental questions unanswered. Can the auditory spatial tuning of tectal units be altered by similar manipulations in adult animals? Can altered spatial tuning return to normal if the earplug is removed before the owl reaches adulthood? Does the change in spatial tuning that is observed in the optic tectum represent a tectal process or does it reflect changes occurring at lower levels in the auditory pathway? These issues are addressed in this report.

The present study demonstrates striking parallels between experience-dependent changes in the spatial tuning of auditory units in the tectum and previously reported changes in sound localization behavior. This does not mean that the tectum is involved in the perception of sound location. The physiological-behavioral parallels simply indicate that the same, or equivalent, changes affect both processes. Anatomical experiments presented here demonstrate that alterations in spatial tuning measured in the optic tectum reflect modifications occurring at earlier stages in the auditory pathway, at or before the level of the inferior colliculus.

\section{Materials and Methods}

Monaural plugs were used to alter auditory experience. The choice of ages for earplug insertion and removal was based on the results of behavioral expcriments on the effects of monaural occlusion on sound localization (Knudsen et al., 1984a, b). In those previous experiments, barn owls were monaurally occluded at various ages. Animals that were monaurally occluded before 8 weeks of aye learned to inlerpret correctly the abnomal cues induced by the earplug and developed accurate sound localization; animals occluded at later ages did not and retained localization errors. When earplugs were removed from young owis that had adjusted to the abnormal cues, localization errors (in the opposite direction from the original errors) were exhibited that disappeared over a period of weeks. However, when birds were raised to 7 to 8 months of age before removing the earplug, the localization errors that appeared upon earplug removal persisted. Because of these results, most of the birds in this study were monaurally occluded before 6 weeks of age and remained plugged until past the age of 7 months.

Chronic monaural occlusion was accomplished by suturing a dense, foam rubber plug (E. A. R. Cabot Corp.) into the external meatus while the animal was anesthetized with halothane and nitrous oxide. The schedules of earplugging are given in Table 1 . The acoustic effects of the earplugs were not measured in these birds because implanting electrodes for cochlear microphonic recording can aiter sound transmission to the eardrum and can damage the cochlea, which would render subsequent neurophysiological data uninterpretable. However, the effects of such earplugs were measured as part of another study (Knudsen et al,, 1984a). Based on cochlear microphonic recordings from six ears in three owls, a freshly inserted earplug

TABLE I

Audilory histories of experimental birds

\begin{tabular}{ccccc}
\hline Owl & $\begin{array}{c}\text { Ear } \\
\text { Plugged }\end{array}$ & $\begin{array}{c}\text { Earplug } \\
\text { Inserted } \\
\text { (days old) }\end{array}$ & $\begin{array}{c}\text { Earplug } \\
\text { Removed } \\
\text { (days old) }\end{array}$ & $\begin{array}{c}\text { Period of } \\
\text { Recording } \\
\text { (days old) }\end{array}$ \\
\hline 1 & left & 33 & 150 & $132-150$ \\
2 & right & 38 & 170 & $153-191$ \\
3 & right & 31 & n.r. & $161-186$ \\
4 & right & 43 & 202 & $328-359$ \\
5 & right & 31 & 261 & $250-285$ \\
6 & left & 41 & 342 & $335-348$ \\
7 & right & 30 & 360 & $685-734$ \\
8 & left & 56 & 385 & $377-385$ \\
9 & left & 250 & 613 & $585-643$ \\
\hline
\end{tabular}

${ }^{a}$ n.r. = earplug was never removed. attenuates sound by 17 to $53 \mathrm{~dB}$ from 2 to $10 \mathrm{kHz}$ and delays sound by 0 to $80 \mu \mathrm{sec}$ over a range of 3 to $10 \mathrm{kHz}$. I he effect of the earplug varies with frequency and from one ear to the next. Moreover, because the birds in this study were occluded for periods of up to 1 year, the acoustic properties of the earplugs undoubtedly changed somewhat due to stretching of the ear canal, accumulation of earwax, and hardening of the foam rubber. Thus, these values must be regarded as approximations. However, the earplugs remained firm and complete throughout the experiments, and the elevated unit thresholds with earplugs in place and the shifts in unit spatial tuning upon earplug removal demonstrate clearly that the earplugs were effective.

Physiology. Neurophysiological recordings were made from the optic tecta of 12 barn owls (Tyto alba): 9 owls were subjected to monaural occlusion during various stages of development, and 3 owls were raised without earplugs and served as controls. Data from 3 of the monaurally occluded animals (owls 4,5 , and 6) and 2 of the control animals have appeared in other publications (Knudsen, 1983a, 1984a). In most cases, recordings were made from both tecta over a period of weeks or months. Data were gathered before and after removal of the earplug in all cases except for owls 3, 4, and 7 (Table I); owl 3 was recorded from only while the earplug was in place and owls 4 and 7 were recorded from only long after the earplugs were removed.

The methods of unit recording, stimulus presentation, and data analysis were the same as those used in previous studies (Knudsen, 1982, 1984a). All surgical procedures were performed with the owls anesthetized with halothane and nitrous oxide; stainless steel recording chambers and bolts for securing the head during recording sessions were implanted. On the day of an experiment, the animal was anesthetized with an intramuscular injection of ketamine hydrochloride $(10 \mathrm{mg} / \mathrm{kg})$ and diazepam $(2 \mathrm{mg} / \mathrm{kg})$. Anesthesia was maintained with a constant infusion of ketamine hydrochloride $(5 \mathrm{mg} /$ hr) delivered into the breast muscle. The anesthetized bird was wrapped in a leather harness and suspended in a prone position inside a sound isolation chamber equipped with a remotely controlled, movable speaker. The speaker moved on an imaginary sphere centered on the owl's head and at a distance of $92 \mathrm{~cm}$. The owl's head was positioned using retinal landmarks (the eyes of the barn owl are essentially stationary in the head) so that the median sagittal and visual planes lined up with $0^{\circ}$ azimuth and $0^{\circ}$ elevation, respectively, of the speaker movement system. The locations of auditory and visual stimuli are given in degrees left or right of the median sagittal plane (azimuth) and in degrees above or below the visual plane (elevation) measured at the center of the head. The properties of this coordinate system are discussed in Knudsen (1982).

Acoustic stimuli consisted of noise and tone bursts, $100 \mathrm{msec}$ in duration, presented once per second. Rise and fall times were $2.5 \mathrm{msec}$ for tone bursts and 0 msec for noise bursts. The noise burst stimulus served as the search stimulus as the electrode was advanced through the tectum. Sound field calibrations were carried out with a 0.5 -inch condenser microphone centered in the chamber in place of the owl. Sound pressure levels are root mean square values expressed in decibels relative to $20 \mu \mathrm{Pa}$; noise stimuli were measured using the " $A$ " weighting scale. Sound levels were constant $( \pm 3 \mathrm{~dB})$ from 2 to $12.5 \mathrm{kHz}$ and rolled off at about $20 \mathrm{~dB}$ per octave below and about $6 \mathrm{~dB}$ per octave above this range.

Visulal stimuli consisted of light and dark bars and spots projected onto a translucent hemisphere that was brought into the sound chamber for visual testing and then was removed. The surface of the hemisphere was $57 \mathrm{~cm}$ from the center of the owl's head.

Units were recorded extracellularly with micropipettes or glass-coated tungsten microelectrodes. Electrodes were positioned according to landmarks on the skull and passed through the telencephalon for aboul $1.5 \mathrm{~cm}$ before reaching the optic tectum. The appearance of "bursty" unit activity sensitive to visual and auditory stimulation signaled the entrance of the electrode into the superticial tectal layers (Knudsen, 1982, 1984a). When tungsten microelectrodes were used, recording sites were marked with electrolytic lesions and were reconstructed later in transverse, Nissl-stained sections. Unit activity was monitored on an oscilloscope and an audio monitor. The output of a level discriminator was sent to an event counter and a raster generator for on-line analysis of stimulus-locked responses. The activity of selected units was recorded on magnetic tape for off-line analysis.

Best areas. The threshold of a unit to noise bursts was determined with the speaker within the best area. The unit's responses to eight presentations of the noise stimulus at $20 \mathrm{~dB}$ above threshold were then measured every 2.5 or $5^{\circ}$ in azimuth and every 5 or $10^{\circ}$ in elevation across the unit's receptive field. Borders of best areas were defined as those speaker locations where the noise stimulus elicited half of the maximum number of spikes for that stimulus. When a border fell between two sampled points, it was approximated by linear interpolation. The geometric center of these borders indicated the center of the best area. 
Latencies. The time lag between the stimulus gate pulse and the onset of the cochlear microphonic (measured previously) was $3 \mathrm{msec}$. This sound propagation time was subtracted from the latencies of all units measured relative to the gate pulse. Thus, the latencies reported here indicate unit latencies relative to the onset of the cochlear microphonic.

Frequency response properties. The frequency to which the unit was most sensitive was its characteristic frequency (CF). The range of frequencies to which a unit responded at $10 \mathrm{~dB}$ above its CF threshold was measured by setting the stimulus intensity at the appropriate level and increasing or decreasing the frequency until the unit responded. These high and low frequency "cutoffs" were used to calculate $Q$ values, a measure of the sharpness of frequency tuning. Frequency selectivity values ( $Q$ values) were calculated by dividing the CF of a unit by the range of its frequency sensitivity at $10 \mathrm{~dB}$ above $\mathrm{CF}$ threshold

Modality balance. To represent the relative effectiveness of visual versus auditory stimuli in driving units, a modality balance index was developed. Units were classified into one of eight categories based on the vigor of their responses to auditory versus visual stimuli when the stimulus of each modality was presented alone: 0 , not driven by either visual or auditory stimuli; 1 , driven by visual stimuli only; 2 , driven by visual stimuli and driven weakly by auditory stimuli (auditory receptive field not measurable); 3 , driven much more strongly by visual than by auditory stimuli; 4 , driven approximately equally by visual and auditory stimuli; 5 , driven much more strongly by auditory than by visual stimuli; 6 , driven by auditory stimuli and driven weakly by visual stimuli (visual receptive field not measurable); 7 , driven by auditory stimuli only.

This classification scheme was neither quantitative nor comprehensive, yet it provided some assessment of the responsiveness of units to auditory and visual stimulation. The prevalence of different categories of bimodal types was certainly affected by the limitations of the stimuli and by the usc of a repetitive, auditory stimulus as the search stimulus when advancing the electrode. Particularly, the proportion of visual-only (category 1) units is lower than would be expected had a visual search stimulus been used. Thus, although the distribution of bimodal types are comparable across animals within this study, they should not be compared quantitatively with data from other studies.

Bimodal fraction. In order to represent modality balance profiles as a single number, a "bimodal fraction" was computed. This was the number of clearly bimodal units (categories 3,4 , and 5 ), divided by the total number of units assayed. Thus, a bimodal fraction of 1 indicates that all units were bimodal, whereas a fraction of 0 indicates that no units could be driven well by both types of stimuli.

Anatomy. Marking lesions were made by passing $10 \mu \mathrm{A}$ of cathodal current for 10 seconds. In one owl (owl 4), horseradish peroxidase (HRP) ( $20 \%$ in Tris buffer, $\mathrm{pH} 8.3$ ) was injected iontophoretically into both tecta. After $30 \mathrm{hr}$ survival time, the owl was deeply anesthetized with sodium thiopental, and the brain was perfused, sectioned, and reacted for HRP with tetramethyl benzidine according to the procedure described by Knudsen and Knudsen (1983).

Behavior. Two owls (owls 4 and 5) were trained to turn their heads toward sound sources for our behavioral assay of sound localization (Knudsen et al., 1982, 1984a, b). Details of the training and testing procedures are given in Knudsen et al. (1984a). Briefly, auditory (noise bursts) and visual (lightemitting diode) targets were presented at random locations in a darkened sound chamber. The owl was required to respond by attempting to face the target. The owl was rewarded if it reacted to the stimulus with a head movement, whether it oriented accurately or not. Because owls have a natural tendency to face auditory and visual stimuli, this task was learned quickly. Final head orientation, indicated by a reflected infrared beam, was measured relative to the location of the target. On each day, mean values of responses to the visual stimulus and to the auditory stimulus were computed. A difference between these means was interpreted as an error in sound localization.

\section{Results}

The following unit properties were quantified in control birds and in experimental birds before and after removing the earplug: (1) modality balance, (2) threshold sensitivity to noise bursts, (3) minimum latency, (4) frequency tuning. (5) size of auditory receptive field, and (6) alignment of auditory best area center with visual receptive field center. It has been demonstrated previously that these properties, with the exception of modality balance, vary topographically in the tectum (Knudsen, 1984a). Therefore, in order to control for differences due to recording site, the properties of units
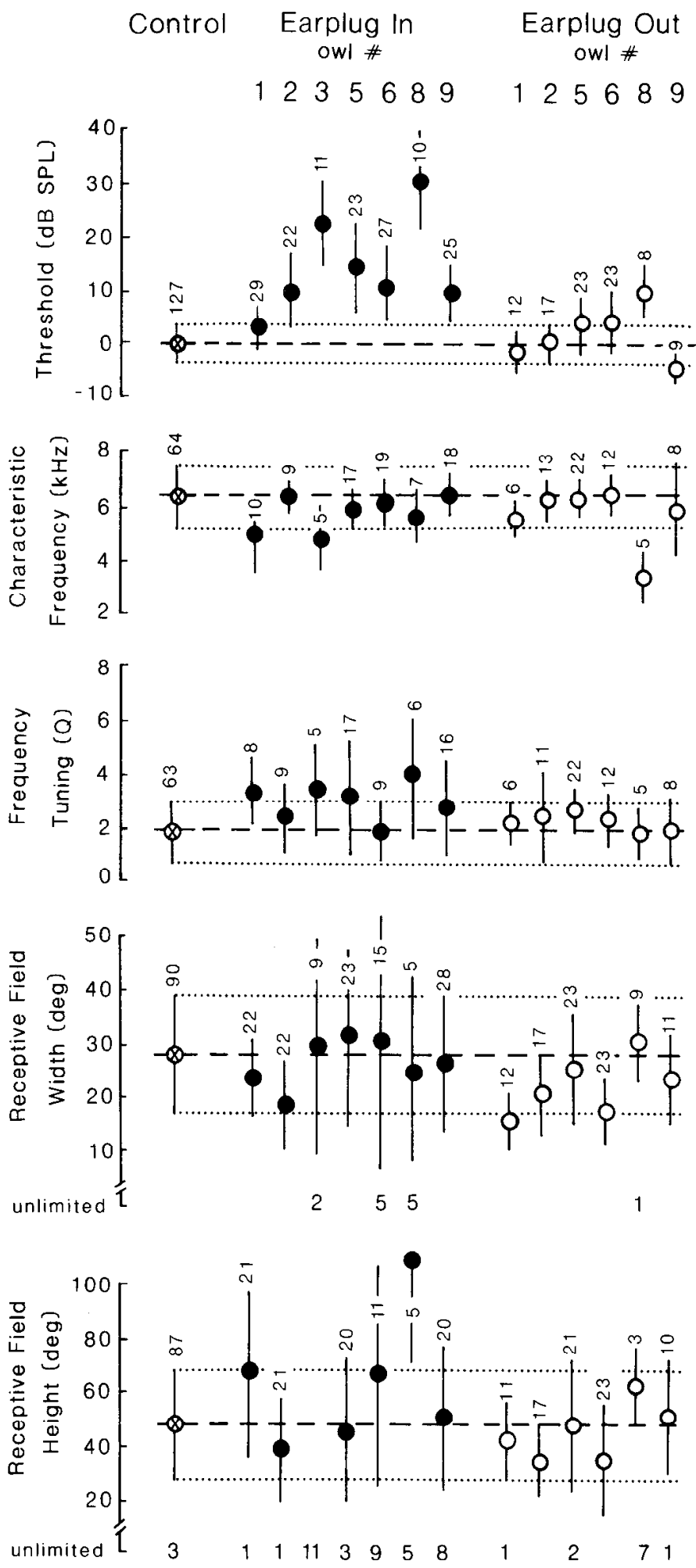

Figure 1. Unit response properties measured before and soon after earplug removal. Circles represent mean values, vertical lines indicate standard deviations of the sample, and the associated numbers are the sample sizes. The identity of the owl is indicated at the top. The horizontal dashed lines mark the mean values of control samples and the dotted lines represent the standard deviations of those samples. The data are from units in the rostral tectum with visual receptive fields within the frontal area of space (see "Results"). Control data $(\otimes)$ were compiled from three birds. Solid circles represent data collected with the earplug in place for at least 4 months. Open circles represent data collected within 1 week after earplug removal. The numbers of units that had unlimited auditory rocoptive ficlds in a particular dimension are indicated in the bottom two plots. 
A

No. of Spikes

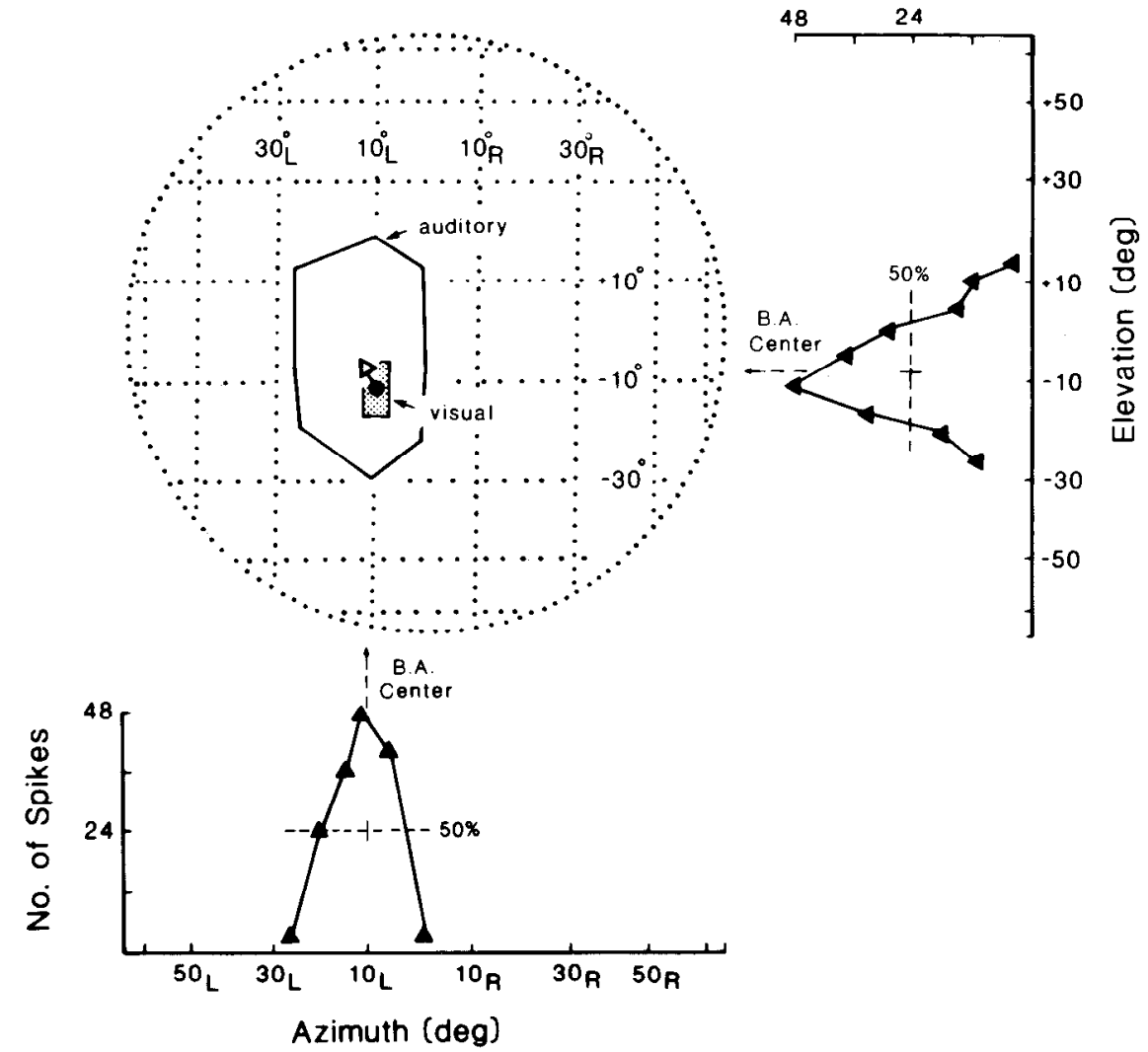

B

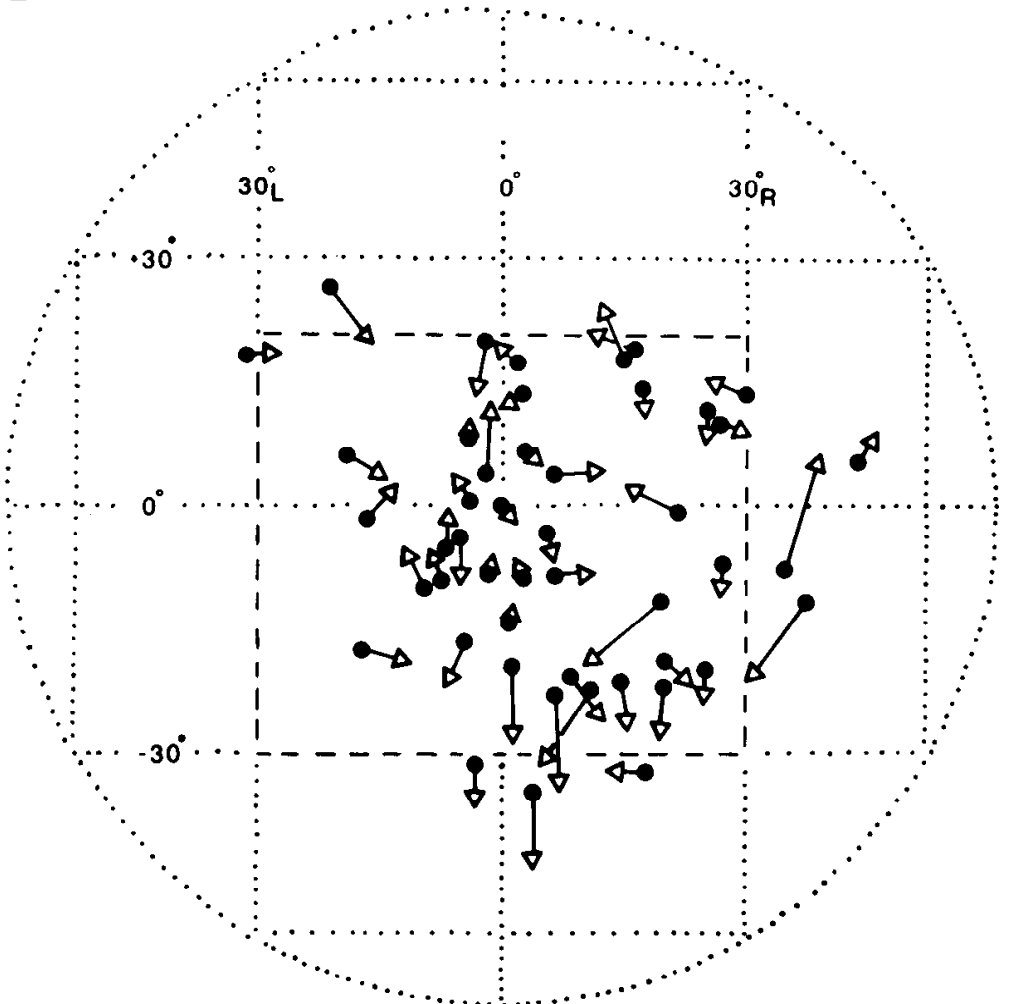

C

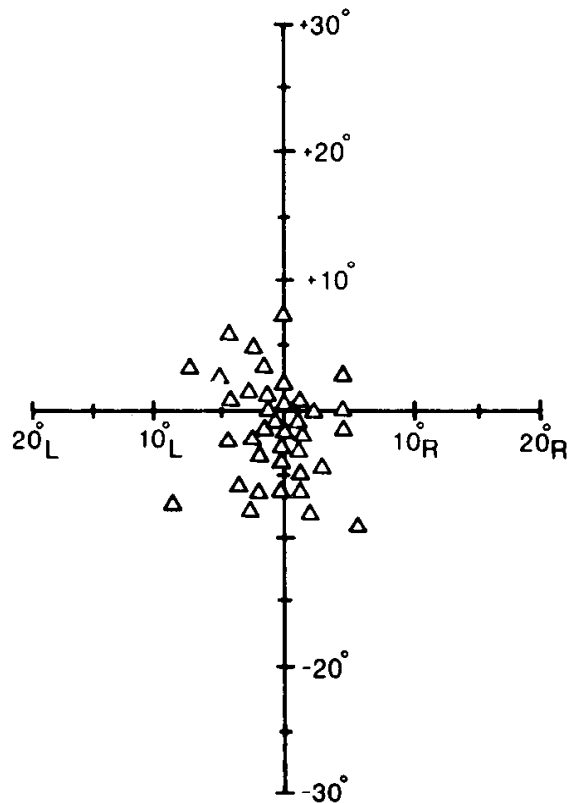

Figure 2. The alignment of auditory best areas with visual receptive fields in one control animal. In $A$, the auditory spatial tuning and visual receptive field of a single unit are plotted on a dotted globe of space. Below and to the right arc the responses of the unit to eight presentations at each location of a noise burst $20 \mathrm{~dB}$ above the unit's threshold. Locations from which the noise burst elicited $50 \%$ of the maximum response were used to define the limits of the best area. The geometric center of these limits was the best area center indicated by the open triangle in the field plot. The solid circle indicates the geometric center of the visual receptive field (stippled region). In $B$, the lociations of auditory best area centers $(\triangle)$ relative to visual receptive field centers (-) for 47 single units are plotted on coordinates of space. Lines connect data from an individual unit. The response properties of units with visual receptive fields centered within the dashed rectangle were compared between owls (e.g, Fig. 1). In $C$, the locations of auditory best area centers relative to visual receptive field centers are plotted for 38 units that had visual receptive tields centered within the frontal region (rectangle in $B$ ). $L$, left; $R$, right; $B$. $A$., auditory best area. 
from a restricted, rostral region of the tectum were compared across animals. The region of tectum chosen for comparison was defined by the visual representation of $30^{\circ}$ left to $30^{\circ}$ right and $20^{\circ}$ up to $30^{\circ}$ down. In normal owis, units with visual receptive fields centered within this range exhibit little systematic variation in their response properties (Knudsen, 1984a).

Among the experimental birds (Table I), the data from owl 9 (the only animal to be monaurally occluded as an adult) were qualitatively different from those collected from owis 1 to 8 . For this reason, the data from owl 9 are presented separately at the end of "Results" (Fig. 12).

\section{Control animals}

The response properties of units in the optic tectum of control owls have been described in detail in previous reports (Knudsen, 1982, 1984a). The following statements pertain to those units in the rostral portion of the tectum that will be compared across animals (Fig. 1). Units did not habituate to repetitive stimulation, although fluctuations in excitability were frequently observed. Thresholds to noise stimulation were low (mean $=0.2 \mathrm{~dB} S P L$ ). Response latencies ranged from 6 to $160 \mathrm{msec}$, with a median value of $15 \mathrm{msec}$. Frequency tuning typically was broad (mean $\mathrm{Q}=1.9 \pm 1.2 \mathrm{SD}$ ) with poorly defined CFs that were toward the upper end of the owl's audible range (mean $\mathrm{CF}=6.5 \mathrm{kHz} \pm 1.0$ ). Auditory spatial fields ranged in size from $8^{\circ}$ to $55^{\circ}$ (mean $=28^{\circ}$ ) in width and from $10^{\circ}$ to unbounded in height. Of 90 units, 87 had receptive fields with upper and lower boundaries; the mean height of these fields was $48^{\circ}$. The fields of 2 units had lower but no upper boundaries and the field of 1 unit had no boundaries in elevation. The alignment of auditory best areas with visual receptive fields was consistently good in all 3 control animals: the directions of the misalignments were approximalely random (Fig. 2) and the magnitude of the average alignment error was $3.4^{\circ}$.

Throughout most layers of the tectum, units tended to respond strongly to visual and auditory stimuli. The average bimodal fraction (see "Materials and Methods") for all units encountered was 0.65
(Fig. 3). Visual-only units (category 1) were largely restricted to the most superficial layers, and auditory-only units (category 7 ) were restricted to the deepest layers.

The effect of acute monaural occlusion on auditory response properties in control animals was tested on 12 units located in the rostral tectum. The effect was always the same: monaural occlusion abolished auditory responses regardless of the location of the source or the level of the sound (up to $70 \mathrm{~dB} \mathrm{SPL}$ ). Because the units became unresponsive, the immediate effect of occlusion on unit thresholds and frequency tuning could not be determined.

\section{Effects of early monaural occlusion}

Of the eight animals that were chronically occluded on or before 56 days of age, six were recorded from while the earplug was in place (Table I). By the time the recordings were made, these birds had been earplugged for 99 to 321 days. In all of these birds, units occasionally responded sluggishly or habituated to repetitive acoustic stimulation, and in some portions of the tectum no auditory activity could be recorded. Bimodal interaction was depressed: bimodal fractions ranged from 0.10 to 0.46 , indicating that most units could be driven either by visual or by auditory stimuli, but usually not by both (Fig. 3). In addition, thresholds to auditory stimulation were elevated relative to those in control birds (Fig. 1): the increase was small in owl 1 (mean $=3.6 \mathrm{~dB}$ ) but was substantial $(10$ to $30 \mathrm{~dB}$ ) in the other five birds. (The increase in unit thresholds can be considered an indirect indicator of the effectiveness of the earplug in each bird.)

Minimum response latencies and characteristic frequencies were normal. However, frequency selectivity (Q) tended to be slightly sharper (Fig. 1). This was due to: (1) an increase in low frequency cutoffs from a mean of $4.3 \mathrm{kHz}(n=63)$ in controls to a mean of $4.9 \mathrm{kHz}(n=54)$ in earplugged birds; and (2) a decrease in high frequency cutoffs from a mean of $8.1 \mathrm{kHz}$ to a mean of $7.1 \mathrm{kHz}$.

Sizes of auditory fields were affected differently in different birds (Fig. 1). For the most part, receptive field dimensions remained about normal. The most consistent change was an elongation in
A

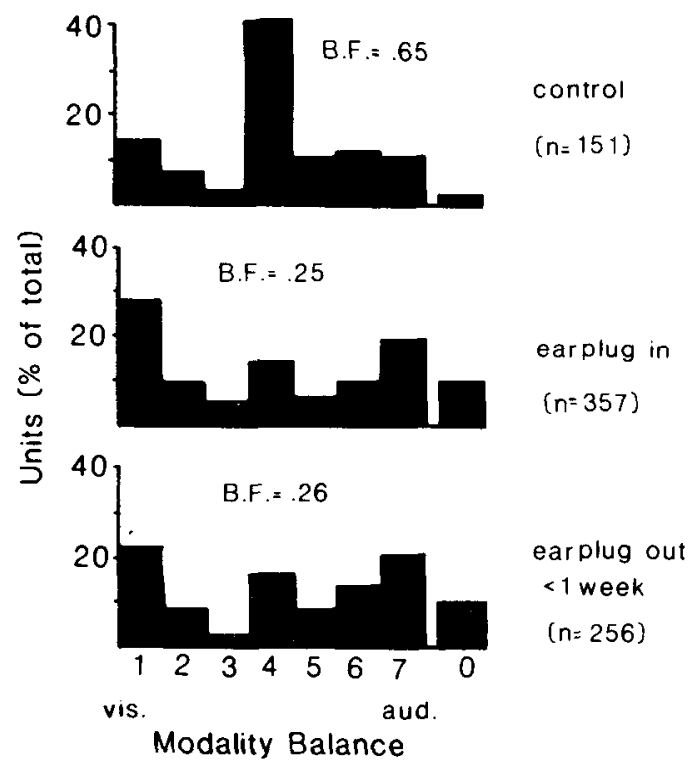

B

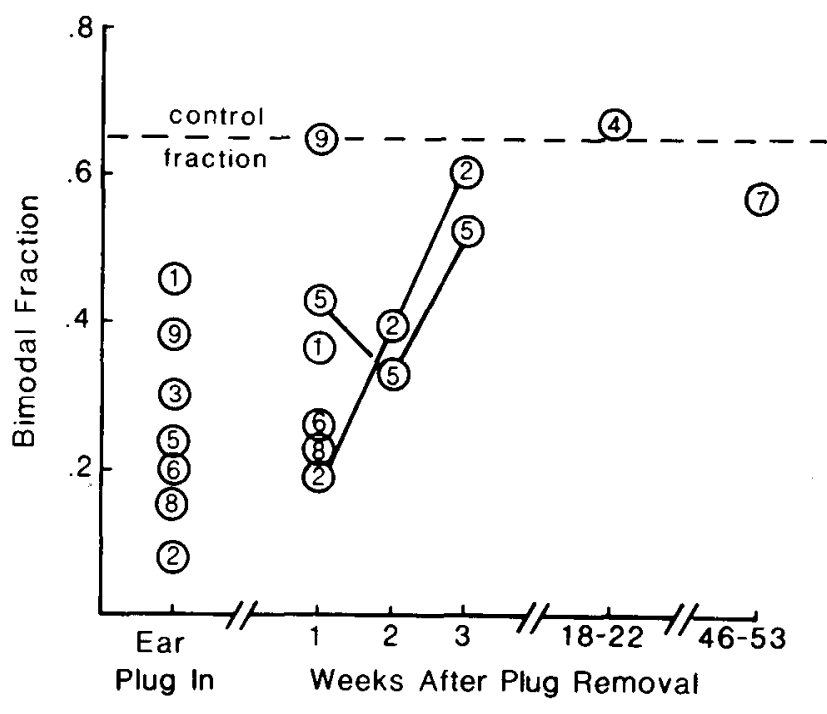

Figure 3. Degree of bimodal responsiveness of auditory-visual units in the tecta of control and experimental owls. In $A$, the histograms represent the

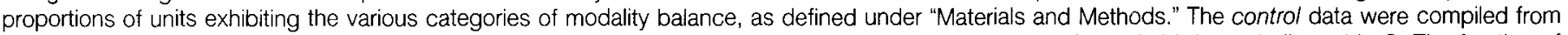

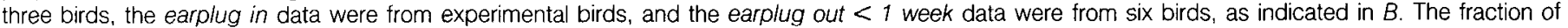

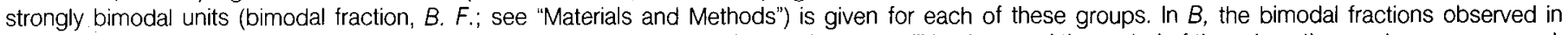

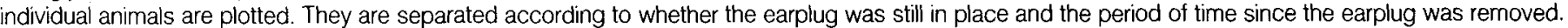

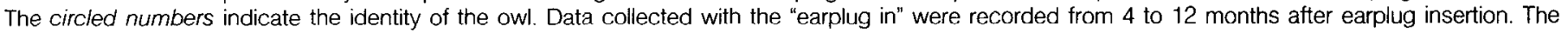
bimodal fraction computed for control birds was 0.65 and is indicated by the dashed line. 
vertical extent observed in owls 1, 3, 6, and 8. Examples of such fields are shown in Figure 4. Not included in the mean values graphed in Figure 1 are receptive fields that were vertically unbounded (indicated as "unlimited"). These accounted for $100 \%$ of the fields in owl $3,45 \%$ of the fields in owl 6 , and $50 \%$ of the fields in owl 8. Two units in owl 3, five units in owl 6, and five units in owl $8 \mathrm{had}$ auditory receptive lields that were unbounded in width as well as in height (e.g., Fig. 4D). Even among these units that exhibited degraded spatial selectivities, the area of space from which an auditory stimulus elicited the greatest number of spikes was usually near to the visual receptive field (Fig. 4).
The magnitude of the average misalignment between auditory best areas and visual receptive fields was slightly greater than it was in control animals: the median was $5.3^{\circ}$, and the range was $1.3^{\circ}$ (owl 1) to $6.9^{\circ}$ (owl 8). The direction of the average misalignment correlated with the ear that was plugged: when the right ear was plugged (owls 2, 3, and 5), auditory best areas tended to be located above visual receptive field centers; when the left ear was plugged (owls 6 and 8), auditory best areas tended to be located below visual receptive fields (Figs. 5 and $7 A$ ). In owl 1, auditory best areas were distributed randomly relative to visual field centers.

Effects of earplug removal. In one owl (owl 6), the properties of a
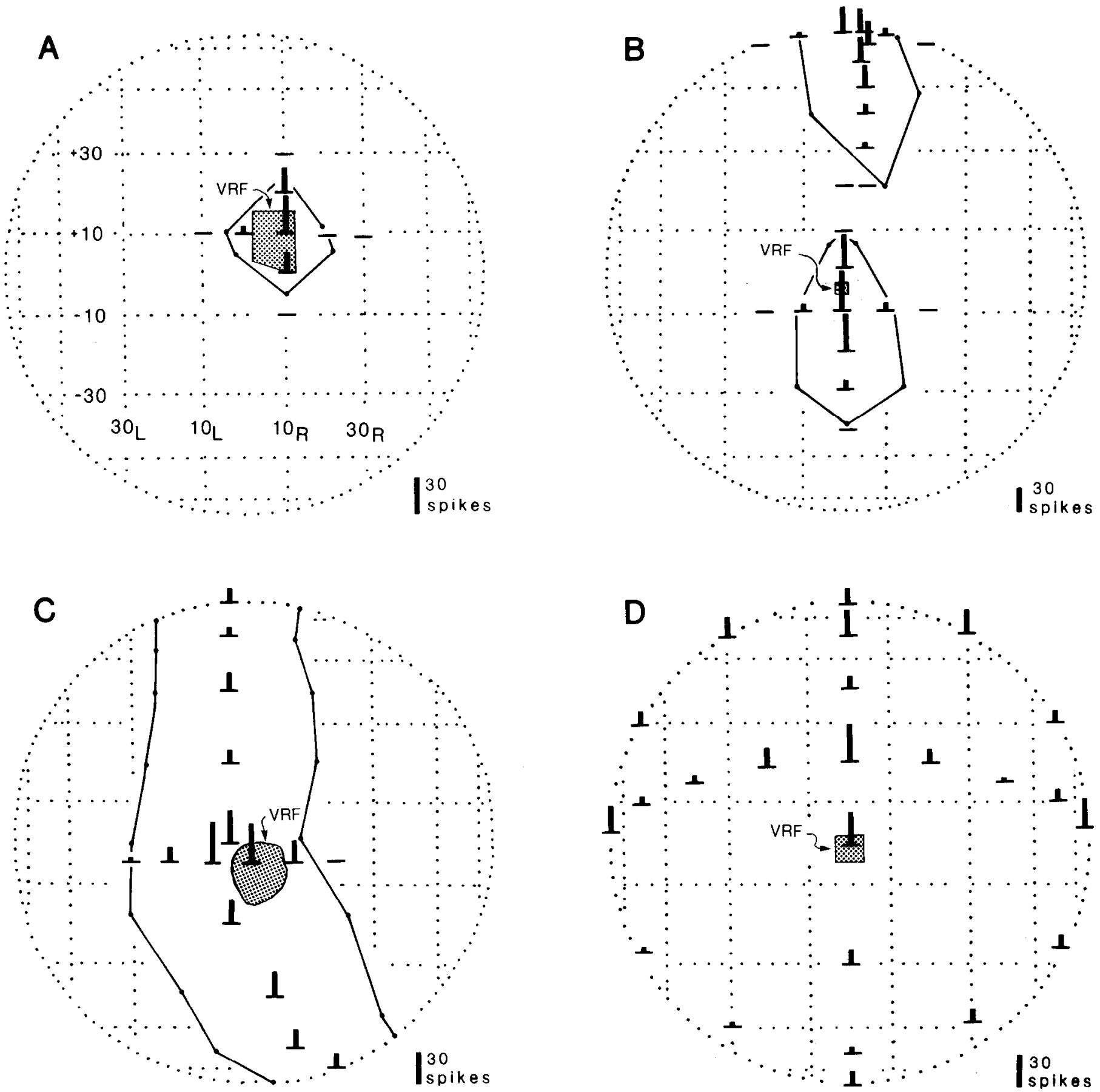

Figure 4. Representative auditory spatial tuning of units in owls with earplugs in place. Plotted onto coordinates of space are the auditory (solid lines) and visual (stippled) receptive fields of four different units. The bars represent the strength of the unit's response to eight noise bursts presented at the location indicated by the base of each bar. The noise bursts were $20 \mathrm{~dB}$ above unit threshold. The data in $A$ are from owl 2; the maximum bar equals 34 spikes. The data in $B$ are from owl 6 ; the maximum bar length equals 50 spikes. The data in $C$ and $D$ are from owl 3 ; the maximum bar lengths equal 35 and 33 spikes, respectively. 

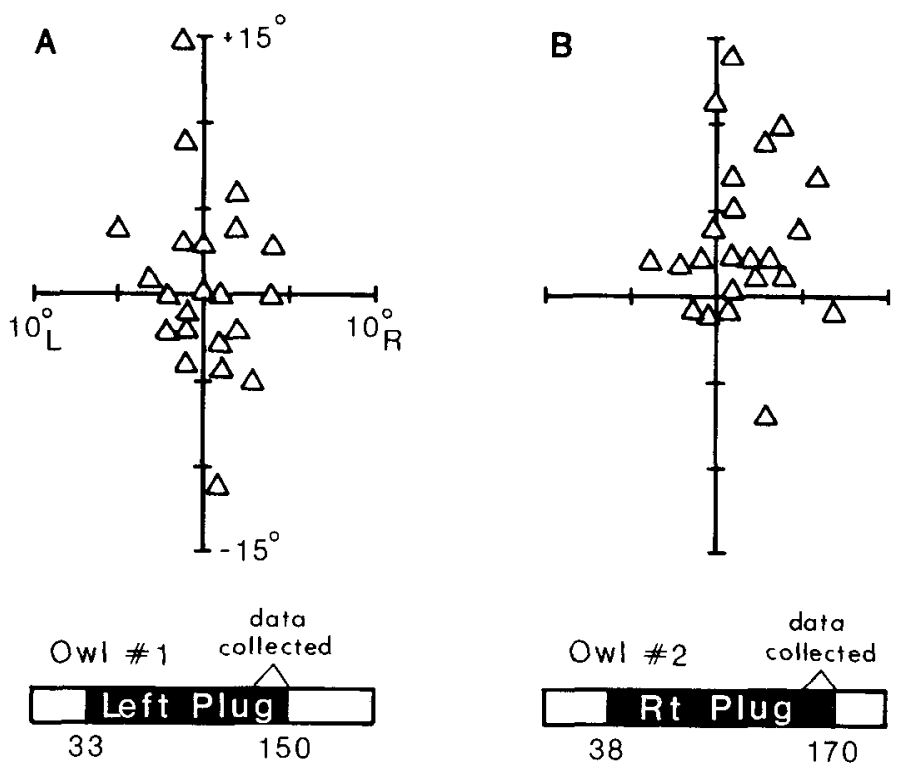

Age (days)

Figure 5. Locations of auditory best area centers relative to visual receptive field centers for units in animals with one ear still occluded. The data in $A$ are from owl 1; the data in $B$ are from owl 2. All of these units had visual receptive fields centered in the frontal area (Fig. 2B). The auditory histories of these birds are indicated at the bottom. Both owls were monaurally occluded early in life.

single unit were characterized before and after removing the earplug. This owl had its left ear plugged at 41 days of age and had the earplug removed at 342 days of age. While the earplug was still in place this unit exhibited the following properties: auditory stimuli were more effective than visual stimuli (category 5); threshold to noise bursts was $10 \mathrm{~dB}$ SPL; response latency was $15 \mathrm{msec}$; CF was $6.2 \mathrm{kHz}$; $\mathrm{Q}$ was 1.4 ; auditory receptive field was $22^{\circ}$ wide by $48^{\circ}$ high; auditory, best area was centered at left $1^{\circ}$ and $-10^{\circ}$, and visual receptive field was centered at left $3^{\circ}$ and $-13^{\circ}$. The unit continued to discharge occasionally as the earplug was removed and had the same height and waveform following earplug removal. Earplug removal did not aller appreciably the unit's modality balance, response latency, $\mathrm{CF}$, or $\mathrm{Q}$. The properties that did change were: its auditory threshold, which decreased by $7 \mathrm{~dB}$; the size of its auditory receptive field, which decreased to $19^{\circ}$ wide by $25^{\circ}$ high; and the location of its auditory best area, which moved right by $9^{\circ}$ and up by $18^{\circ}$.

In four other birds, single units were characterized with the earplug in place, but after the earplug was removed the units were lost or became masked by other units. However, the data from three of these owls were consistent with those described above for owl 6 . In the fourth case (ow 5 ), with the earplug in place, a category 4 unit was recorded that had a best area centered at left $2^{\circ}$ and $+3^{\circ}$ and a visual receptive field centered at left $3^{\circ}$ and $+1^{\circ}$. After the earplug was removed, multiple units were recorded which had visual receptive fields at the same location as the single unit, but which did not respond to acoustic stimulation from any location regardless of sound level. When the earplug was replaced, these units responded vigorously to acoustic stimulation and exhibited approximately the same auditory receptive field as was measured for the single unit. Apparently, these units were selective for the abnormal auditory cues caused by the earplug. Because of this, they were changed from a category 4 to a category 1 bimodal response type by removing the earplug.

Unit properties immediately after earplug removal. Units were isolated within minutes to hours after removing the earplugs in all but owls 3,4 , and 7 . The properties of these units were indistinguish- able from those of units recorded for up to 1 week after earplug removal; hence, thesc data are combined in Figure 1. The data varied between owls, but some general trends were noted. With the earplug removed, responses to auditory stimulation seemed to be stronger and more reliable than they were with the earplug in place. Still, some units were encountered that habituated or responded sluggishly to auditory stimulation, and regions of apparently unresponsive units (silent tissue) were noted in owl 8. Mean auditory thresholds dropped by 4 to $20 \mathrm{~dB}$ following earplug removal. Thresholds were higher than control values in owl 8 , suggesting the possibility of a residual hearing loss in the previously plugged ear of this bird. Response latencies, frequency selectivity (Q), and CFs were normal except in owl 8 . Auditory receptive fields were of normal widths in owls 2,5 , and 8 but were slightly narrower than normal in owls 1 and 6 . The average height of fields that were bounded in elevation was normal. Bimodal interaction (Fig. 3) remained considerably below control levels following earplug removal; bimodal fractions ranged from 0.19 to 0.43 .

The most obvious abnormality following earplug removal was that auditory best areas no longer aligned with visual receptive fields. The direction of the auditory-visual misalignment reflected the ear that had been occluded. In animals raised with the left ear occluded auditory best areas were located to the right and usually above visual receptive fields (Figs. $6 \mathrm{~A}$ and 7 ). In animals raised with the right ear occluded, best areas were located to the left and usually below visual receptive fields (Figs. $6 B, 8,9,10$, and $11 B$ ).

Changes in spatial tuning following earplug removal. Owls 2 and 5 were recorded from repeatedly over the course of 3 weeks following earplug removal. The misalignment between auditory best areas and visual receptive fields seemed to remain fairly constant in owl 5, which was 261 days old at the time the earplug was removed (see Fig. 10). However, in owl 2, which was 170 days old when its earplug was removed, the auditory-visual misalignment clearly decreased and essentially disappeared over this period of time (Fig 8). The most convincing demonstration of this decrease was the progressive realignment of auditory best areas and visual receptive
A

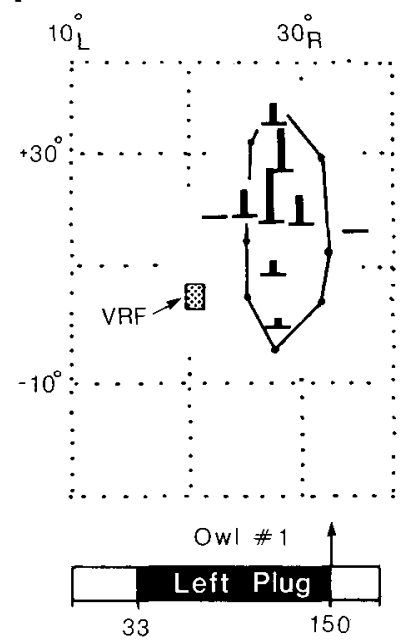

B

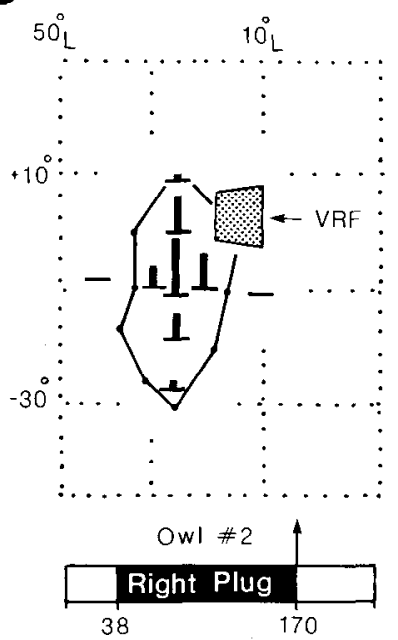

Figure 6 . Shifts in auditory spatial tuning resulting from early monaural occlusion. These are examples of the auditory and visual spatial tuning of bimodal units from owls 1 and 2, both measured on the day the earplug was removed. Auditory (solid lines) and visual (stippled) receptive fields are plotted on coordinates of space. The strength of the units' responses to eight noise bursts at $20 \mathrm{~dB}$ above unit threshold is represented by the heights of the bars, the bases of which indicate the locations of the stimuli, The maximum bar lengths equal 43 spikes in $A$ and 28 spikes in $B$. The auditory history of each bird is represented at the bottom. The upwardpointing arrows indicate when the data were collected. VRF, visual receptive field. 


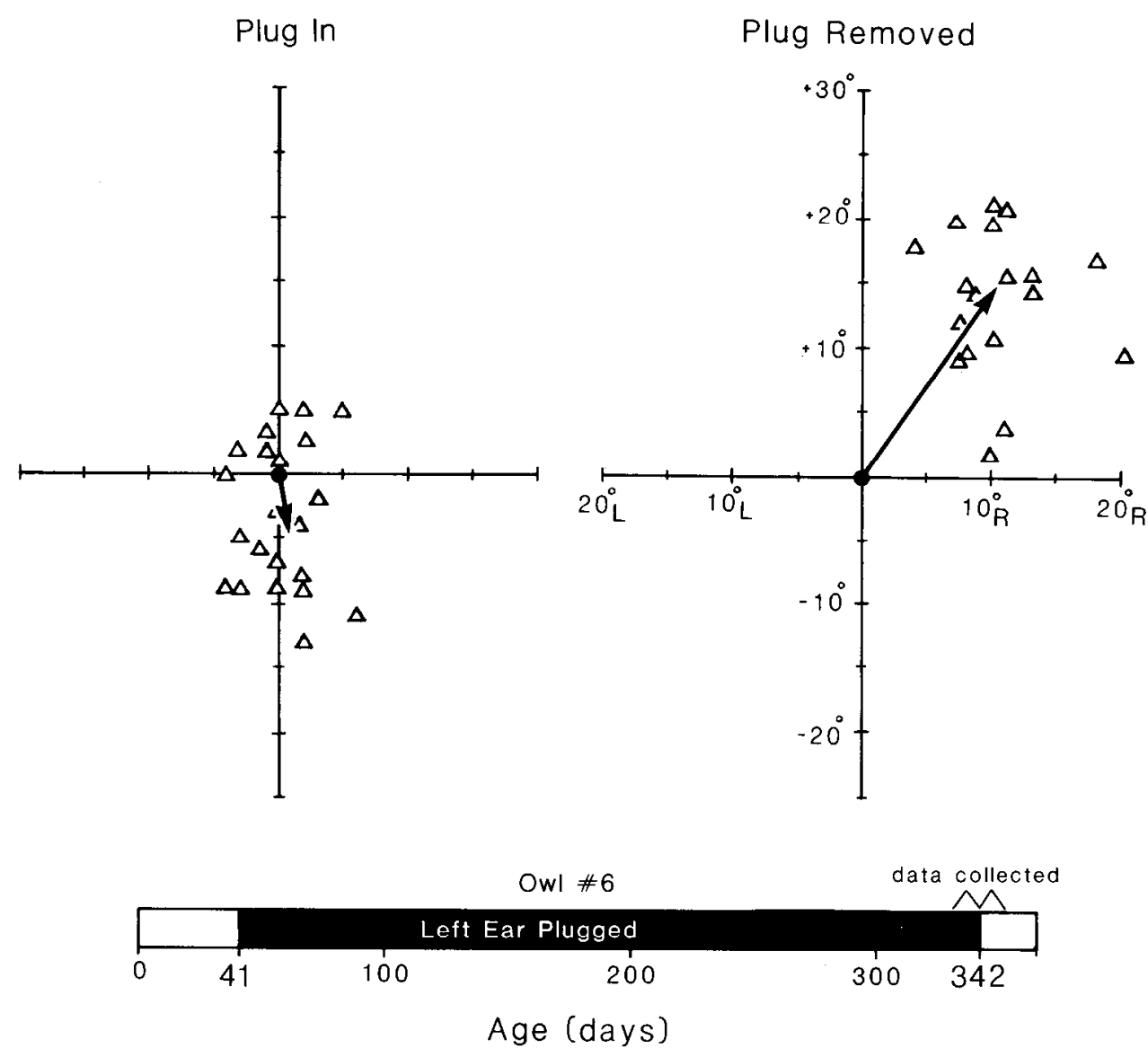

Figure 7. Shifts in the auditory spatial tuning of tectal units in owl 6 which was monaurally occluded at 41 days old. The centers of auditory best areas $(\Delta)$ of all bimodal units located in the rostral tectum are plotted relative to the centers of their visual receptive fields. The vectors represent the median auditory-visual misalignment of these samples. The auditory history of this owl and the period over which the data were collected are indicated at the bottom.

fields of units recorded from approximately the same site in the tectum (Fig. 8A).

Changes in modality balance. During the first 2 weeks following earplug removal, the proportion of units that could be driven strongly by auditory and visual stimuli (categories 3, 4, and 5) in owls 2 and 5 was well below normal (Fig. $3 B$ ). However, by the third week, their bimodal fractions had increased and were approaching normal proportions. In owls 4 and 7 , which had been subjected to monaural occlusion at about the same ages, but which were recorded from long after their earplugs were removed, bimodal fractions were normal. Thus, the apparent decrease in bimodal interaction that resulted from monaural occlusion was still reversible after the animals had reached adulthood.

Long lasting effects of monaural occlusion. Erratic patterns of discharge were occasionally encountered long after the earplug was removed. However, the impressive, lasting abnormality was the clear misalignment between auditory best areas and visual receptive fields that persisted in the animals that had remained monaurally occluded beyond 200 days of age. The data from owls 4 and 7 demonstrate that this misalignment of spatial tuning persisted in adult birds for at least 1 year (Figs. 9 and 11). Units were recorded in owl 4 between 4 and 5 months after earplug removal, and in owl 7 between 11 and 13 months after earplug removal. Because of the amount of time that had elapsed since the removal of the earplugs, it was assumed that auditory spatial tuning had stabilized in these birds, and data collected over a period of weeks and from various portions of the tecta could be combined (Figs. 9 and 11). The pronounced errors in auditory-visual alignment were in the direction expected from the birds having been raised with an occluded right ear: auditory best areas were, in general, located to the left and below visual receptive fields. However, the exact directions and magnitudes of the errors varied markedly across the tecta. The smallest errors were measured in the representation of frontal space, whereas much larger errors were found in the representation of locations in the periphery, parlicularly to the animal's right side. In addition, many units with visual receptive fields located above the plane had auditory best areas at equivalent or higher elevations. Thus, in both birds, early exposure to monaural occlusion had resulted in changes in relative magnification as well as in a shift in the auditory map of space.

Behavioral errors in sound localization. Owls 4 and 5 were tested behaviorally after earplug removal for evidence of sound localization errors (see "Materials and Methods"). Normal owls localize sounds with an accuracy of about $1.5^{\circ}$ in azimuth and elevation (see Fig. $11 A$; Knudsen et al., 1979, 1984a). When tested on three separate days, owl 4 exhibited mean errors of right $9.7^{\circ}$ and up $1.4^{\circ}$ (see Fig. $11 A$ ), right $8.0^{\circ}$ and up $0.3^{\circ}$, and right $8.3^{\circ}$ and down $3.8^{\circ}$. The azimuthal component of this localization error matched approximately the $10.0^{\circ}$ mean leftward shift of auditory best areas relative to visual receptive field centers. However, the tendency of the bird to oricnt at or below the elevation of the sound source was not expected from the generally lower locations of auditory best areas relative to visual receptive fields observed throughout most of the tectum (see Fig. 11B).

Owl 5 also exhibited a marked localization error (Fig. 10). The magnitude and direction of this behavioral crror corrcsponded fairly well to the median auditory-visual misalignment measured physiologically in the tectum on preceding or subsequent days.

The anatomical projection to a shifted auditory map. In order to verify that the auditory map, but not the visual map, had shifted in the tectum, lesions were placed near the auditory and visual repre- 

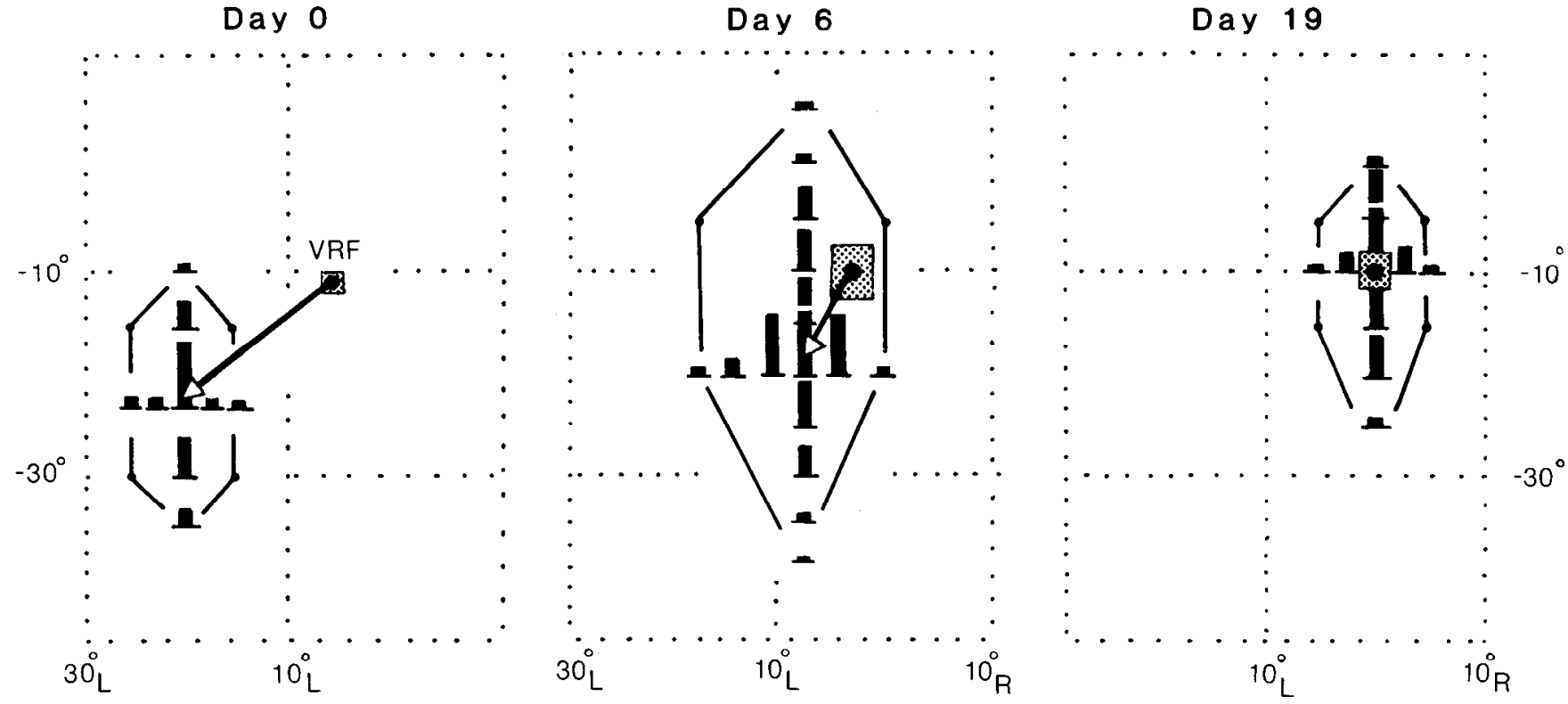

B
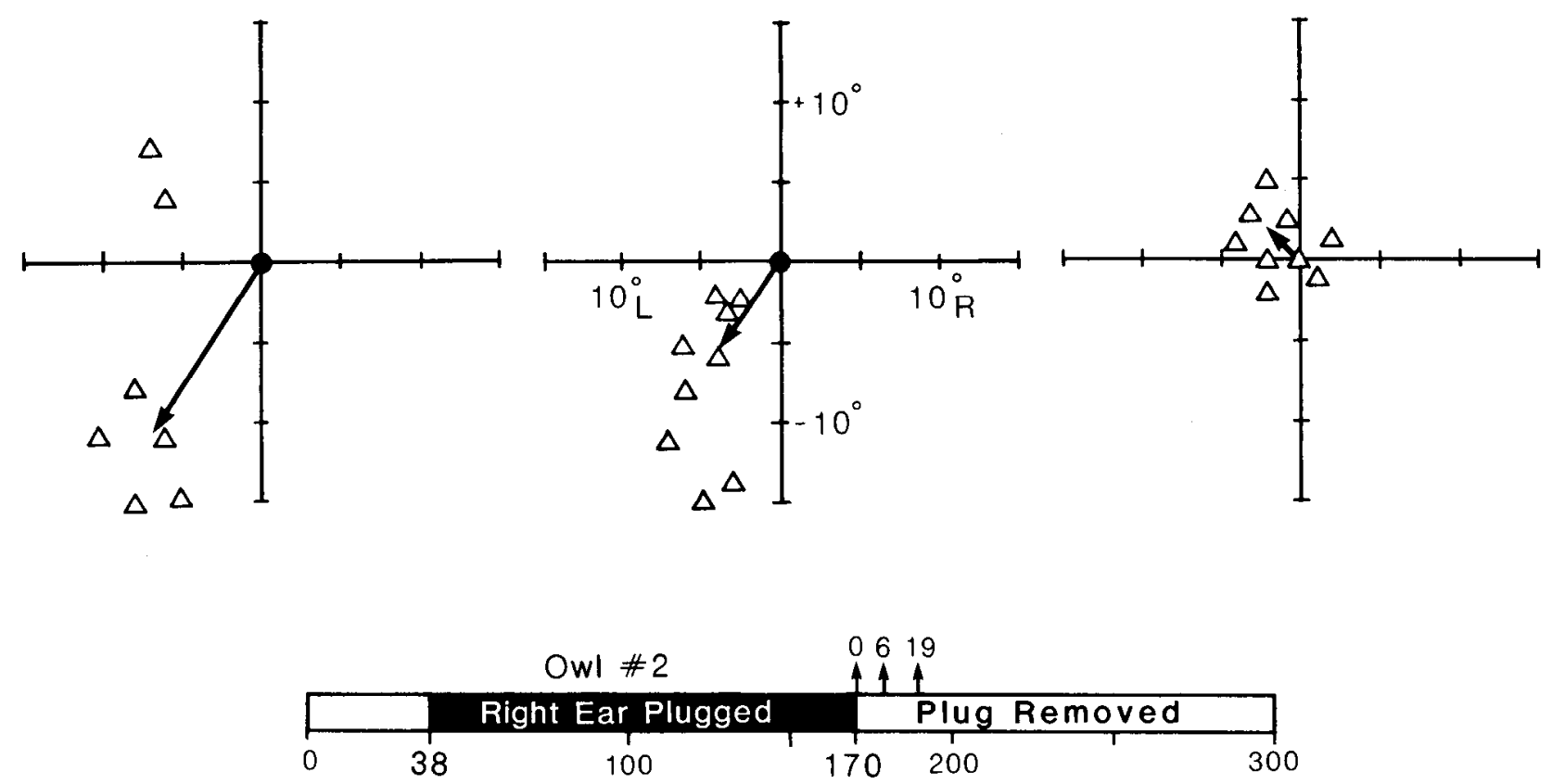

Age (days)

Figure 8. Progressive realignment of auditory spatial tuning with visual receptive fields over time in owl 2 which was unplugged at 170 days of age. In $A$ are plotted the auditory and visual receptive fields of three units which had similarly located visual receptive fields but which were recorded on different days following earplug removal, as indicated above each plot. Ihe open triangles represent auditory best area centers; the solid circles represent visual receptive field centers. Hence, the lines indicate the direction and magnitude of the auditory-visual misalignments. Otherwise, the format is the same as in Figure 6 . In $B$, the locations of auditory best area centers are plotted relative to the locations of visual receptive field centers for all bimodal units in the rostral tectum recorded on the day indicated at the top in $A$. The vectors represent the median auditory-visual misalignments for these samples. The owl's auditory history and the days on which these measurements were made are indicated at the bottom of the figure.

sentations of $0^{\circ}$ azimuth, $0^{\circ}$ elevation (e.g., Fig. 11C). Comparing these lesions with similar lesions placed in control birds (see Knudsen, 1982) showed that the auditory maps had moved in the tecta and the visual maps had remained unchanged. The separation between the lesions indicated that in owl 4 the auditory representation of $0^{\circ}$ azimuth, $0^{\circ}$ elevation had shifted about $1.0 \mathrm{~mm}$ rostrally in the right tectum (measured along the pial surface) and about 1.5 $\mathrm{mm}$ caudally in the left tectum (these values are not adjusted for tissue shrinkage). The shifts of the auditory maps in owl 7 were in the same direction but were slightly smaller: $0.7 \mathrm{~mm}$ in the right tectum and $1.0 \mathrm{~mm}$ in the left.

Is the shift in the auditory space map caused by a shift in the auditory projection to the optic tectum? The auditory space map observed in the optic tectum is generated in the ipsilateral external 


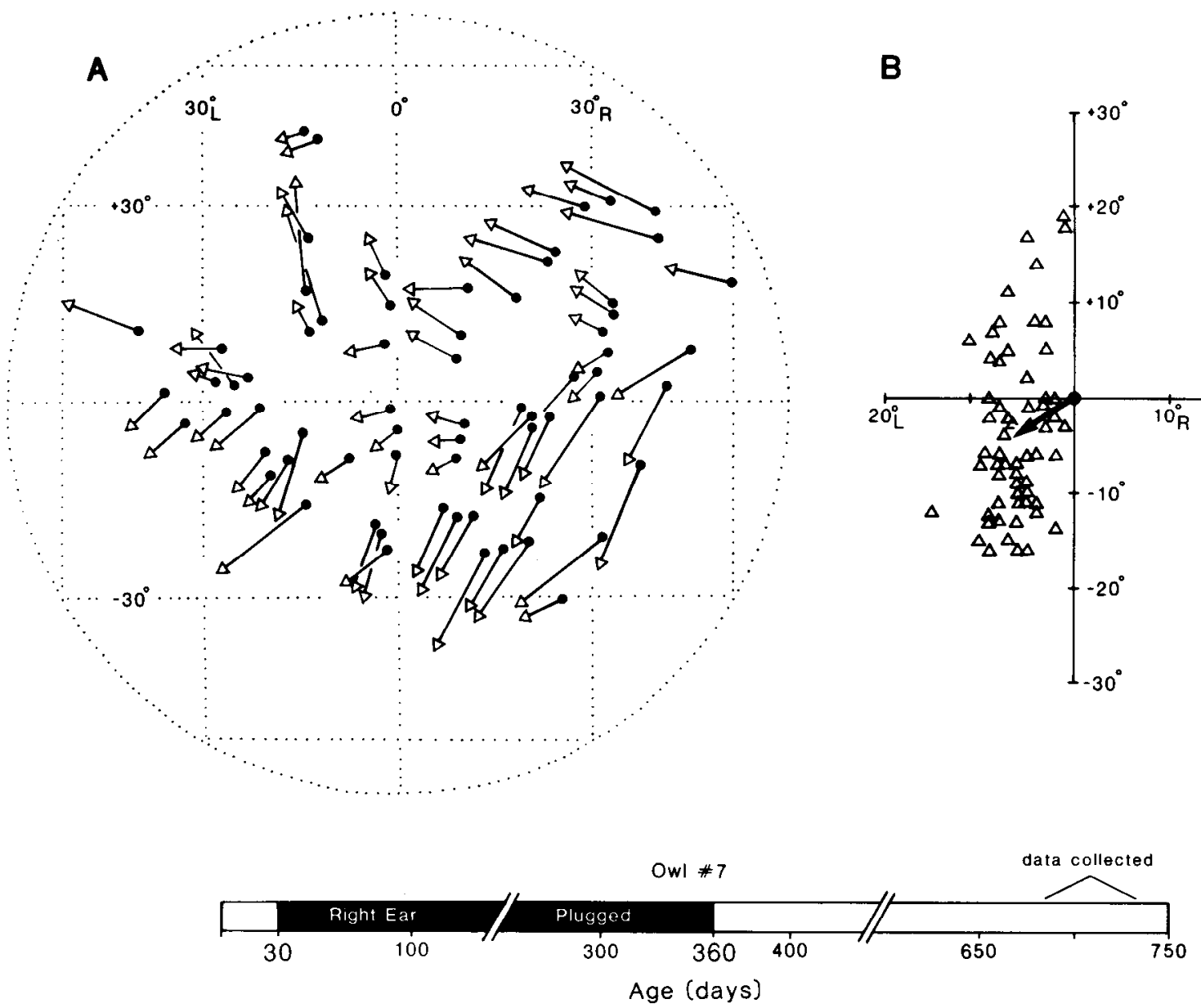

Figure 9. Long-lasting misalignments between auditory best areas and visual receptive fields in an animal that was monaurally occluded until it was an adult. These data from owl 7 were collected over a period of 7 weeks, approximately 1 year after the earplug was removed. In $A$, auditory best area centers $(\Delta)$ and visual receptive field centers $(\Theta)$ are plotted on coordinates of space. The lines connect data from individual units and indicate the direction and magnitude of the misalignments. Not all of the data are shown for units in the rostral tectum, because the overlap of points and lines would have obscured the data. In $B$, best area centers of all bimodal units in the rostral tectum are plottcd relative to the contcrs of their visual receptive fields. The vector indicates the median auditory-visual misalignment for this sample. The auditory history of this owl and the period over which the data were collected are indicated at the bottom.

nucleus of the inferior colliculus $(I C X)$ and is conveyed to the optic tectum by a point-to-point projection (Knudsen and Knudsen, 1983). The ICX is a hooked-shaped nucleus (when viewed in horizontal section), in which the representation of frontal auditory space is located at the rostral, hooked end (Fig. 11C). Because of the large magnification of frontal space in the $\mathrm{ICX}$, and because the representation of $0^{\circ}$ azimuth is located in the middle of the rostral pole of the nucleus, it is easy to distinguish projections that come from what is normally the representation of ipsilateral space (medial corner of the hook) from those that come from the representation of contralateral space (lateral edge of the hook).

The large shift in the representation of auditory azimuth that was found in the tecta of owl 4 provided an opportunity to test for a shifted projection from the ICX (Fig. 11C). One iontophoretic injection of HRP was placed in the right tectum at the representation of auditory left $8^{\circ}$ (contralateral) and $+2^{\circ}$ elevation (which was at visual $0^{\circ}$ azimuth, $+5^{\circ}$ elevation). A second injection was placed in the left tectum at the representation of auditory left $8^{\circ}$ (ipsilateral) and $-1^{\circ}$ elevation (which was at visual right $1^{\circ}$ azimuth, $+2^{\circ}$ elevation). Had the shifted auditory map been due to a shift in the projection from an otherwise normal map in the $\mathrm{ICX}$, then retrogradely filled cells would have been observed along the lateral margin of the ICX on the right side and at the medial corner of the ICX on the left side (indicated by $X$ 's in Fig. 11C). Instcad, the labeled cclls were found in the same relative positions on both sides, clustered in the middle of the rostral pole of the ICX. Thus, the anatomical projection from the ICX to the tectum was apparently normal.

\section{Chronic monaural occlusion of an adult}

Owl 9 was monaurally occluded at 250 days of age (barn owls become sexually mature at about 7 months of age; personal observation). Unit recordings were made beginning 335 days after earplug insertion (al 585 days of age) and were continued until 30 days after earplug removal (at 643 days of age). The 363-day period of monaural occlusion experienced by owl 9 was longer than the period of occlusion experienced by any of the other animals (Table I), yet there were no apparent effects of the monaural occlusion on the response properties of units in the tectum.

With the earplug in place, units that could be driven by acoustic stimuli responded vigorously; sluggish or habituating responses were not observed. Unit thresholds were higher than normal (mean $=9.0$ $\mathrm{dB} S P L$ ), indicating that the earplug was effective. All unit properties were similar to those measured in control animals (Fig. 1), except that the misalignment between auditory best areas and visual receptive fields was extremely large: the average alignment error was $20^{\circ}$ for units in the rostral tectum, and the greatest alignment error was $50^{\circ}$ (Fig. 12A). The direction of the misalignment was that expected from a left ear occlusion: auditory best areas were located to the left 
Physiology
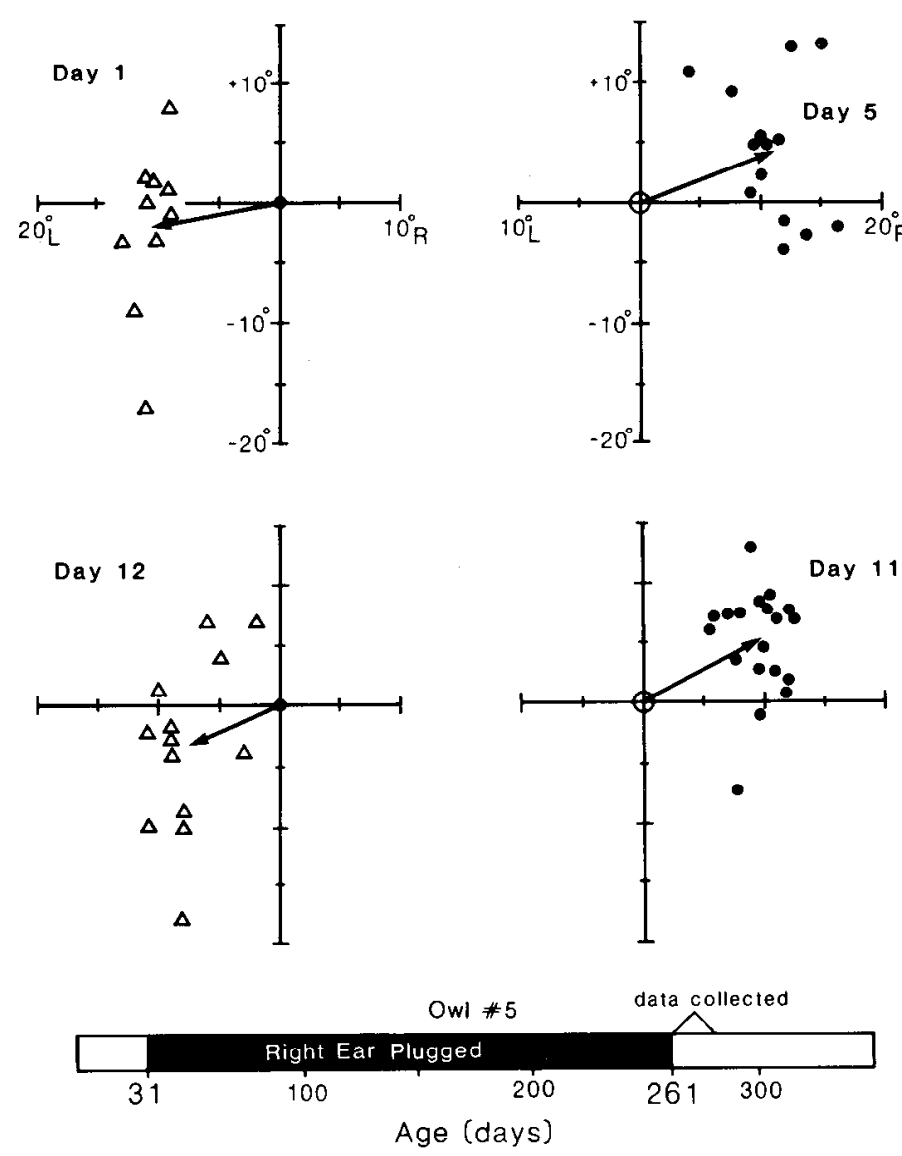

Figure 10. Auditory-visual misalignments measured physiologically compared with sound localization errors measured behaviorally. These data were collected from owl 5 during the 2 weeks immediately following earplug removal, as indicated at the bottom. On the left, the locations of auditory best area centers $(\Delta)$ are plottcd relative to visual rcceptive field centers for all bimodal units recorded in the rostral tectum on the days indicated. The vectors represent the mean misalignments for these samples. On the right, the orienting responses of th is bird lo noise bursts $(\bullet)$ are plotted relative to the location of the speaker (see "Materials and Methods"). The vectors represent the mean localization errors based on these samples. The physiological and behavioral errors are approximately equal in magnitude and opposite direction.

and below visual receptive fields. Units with visual receptive fields located to the bird's left and down (below the dashed line in Fig. $12 A$ ) could not be driven with acoustic stimuli. As a consequence, the number of visual-only units was high, which explains the low bimodal fraction of 0.38 (Fig. $3 B$ ).

Removal of the earplug confirmed that unit response properties had not been altered by the 1-year period of monaural occlusion (Fig. 1). Auditory best areas were aligned well with visual receptive fields (Fig. 12B). Units with visual receptive fields located to the left and down once again responded strongly to acoustic stimuli and exhibited aligned auditory and visual spatial tuning. The bimodal fraction was normal at $0.65(n=62)$

Units throughout much of the tectum in owl 9 responded to acoustic stimulation with the earplug in place. In contrast, the immediate effect of monaural occlusion in control animals was to render unresponsive alf units with frontal auditory fields. Since there was no evidence that the properties of the units in owl 9 had changed, the auditory responsiveness of units in owl 9 with the earplug in place was probably due to a deterioration in the effectiveness of the earplug (see "Materials and Methods").

\section{Discussion}

Sensitive and critical periods in the development of spatial tuning. In animals raised with onc car occluded, the auditory spatial tuning of units in the optic tectum was altered in such a way that, with the ear occluded, the units responded best to sound sources located in approximately the same region of space as their visual receptive fields (Figs. 4, 5, and 7A). Removal of the earplug, which restored a normal balance of sound levels to the ears, caused auditory spatial tuning to move out of alignment with visual receptive fields (Figs. 6 to 11). The direction of the shift in auditory tuning depended on which ear had been occluded: after removal of a left earplug, auditory best areas were located to the right and usually up from visual receptive fields; after removal of a right earplug, they were located to the left and usually down from visual receptive fields.

This experience-dependent change in auditory spatial tuning did not occur in one owl, owl 9 (Fig. 12). The unique feature of this animal's history was that it was subjected to monaural occlusion late in development. Otherwise, the treatment was the same. In fact, the earplug remained in place longer in this animal than in the others, yet the auditory spatial tuning of units showed no evidence of change. With the ear occluded, either auditory responses were absent (for units with low visual receptive fields, Fig. 12A), or best areas were located to the left and below visual receptive fields; after the earplug was removed, best areas were, for the most part, aligned with visual receptive fields (Fig. 12B). When the data from all birds are considered together, they demonstrate that auditory spatial tuning can be altered by abnormal experience, but only during a restricted period early in development.

Realignment of auditory spatial tuning with visual receptive fields following earplug removal also seemed to be age limited. Owl 2 had its earplug removed at 170 days of age, while it was still a juverile. Over the subsequent 3 weeks, the auditory-visual misalignment disappeared (Fig. 8). In contrast, owls 4, 5, and 7 remained monaurally occluded until they were 202, 261, and 360 days old, respectively. In each of these animals, the misalignment between auditory spatial tuning and visual receptive fields seemed stable following earplug removal and persisted for up to 1 year in the case

Figure 11. Behavioral, physiological, and anatomical effects of early and prolonged monaural occlusion. These data were collectcd beginning 4 months after earplug removal from owl 4, which had its right ear occluded from 43 to 202 days of age, as indicated at the bottom. On the left in $A$, the orienting responses of this animal to noise bursts are plotted relative to the location of the speaker (see "Materials and Methods"). The vector represents the mean localization error based on this sample. Data from a control bird are shown on the right for comparison. On the left in $B$, auditory best area centers $(\triangle)$ and visual receptive field centers $(-)$ are plotted on coordinates of space. The lines connect data from individual units and indicate the direction and magnitude of the misalignments. Not all data are shown for units in the rostral tectum, because the overlap of lines and points would have obscured the data. On the right in $B$, best area centers of all bimodal units in the rostral tectum are plotted relative to the centers of their visual receptive tields. The vector indicates the median auditory-visual misalignment for this sample. In $C$, drawings of horizontal sections through the left and right tecta indicate the sites of HRP injections and electrolytic lesions. The lesions were placed at the representations of $0^{\circ}$ azimuth, $0^{\circ}$ elevation in visual space (lesions \#1 and \#4) and in auditory space (lesions \#2 and \#3). The positions of these lesions indicate that the auditory but not the visual maps of space were shifted. The inset is a photograph of the lesions on the left side. The extents of the HRP injection sites (revealed by tetramethyl benzidine processing) are shown by the stippled regions. The auditory and visual spatial tuning measured at the injection sites are indicated. If the auditory spatial tuning were due to a reorganized projection from an unaltered map in the external nucleus of the inferior colliculus (ICX), then retrogradely filled cells (solid dots) should have been found at the sites marked with X's, as shown by Knudsen and Knudsen (1983). ICC, central nucleus of the inferior colliculus; OT, optic tectum. 
A
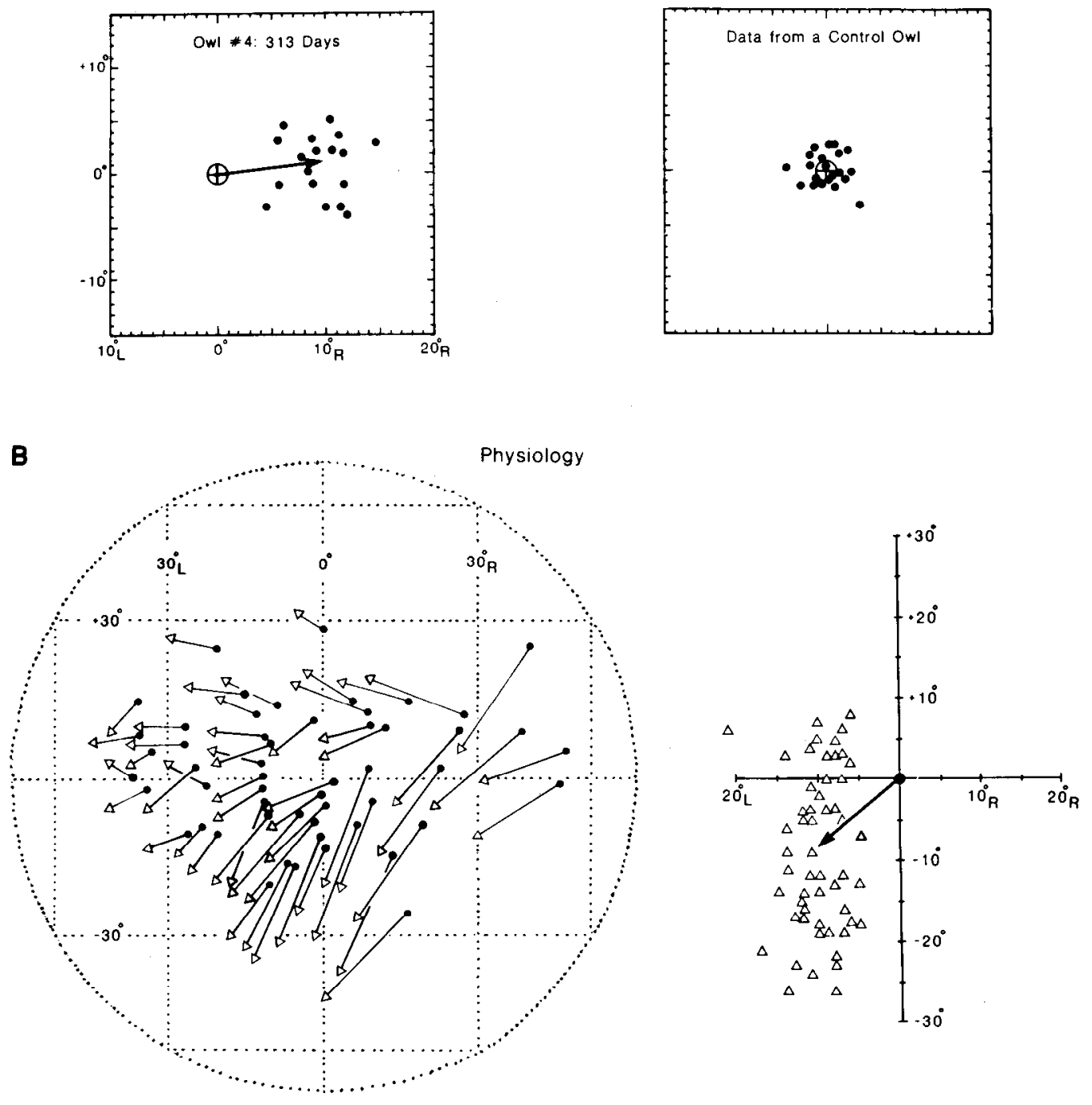

C

Anatomy
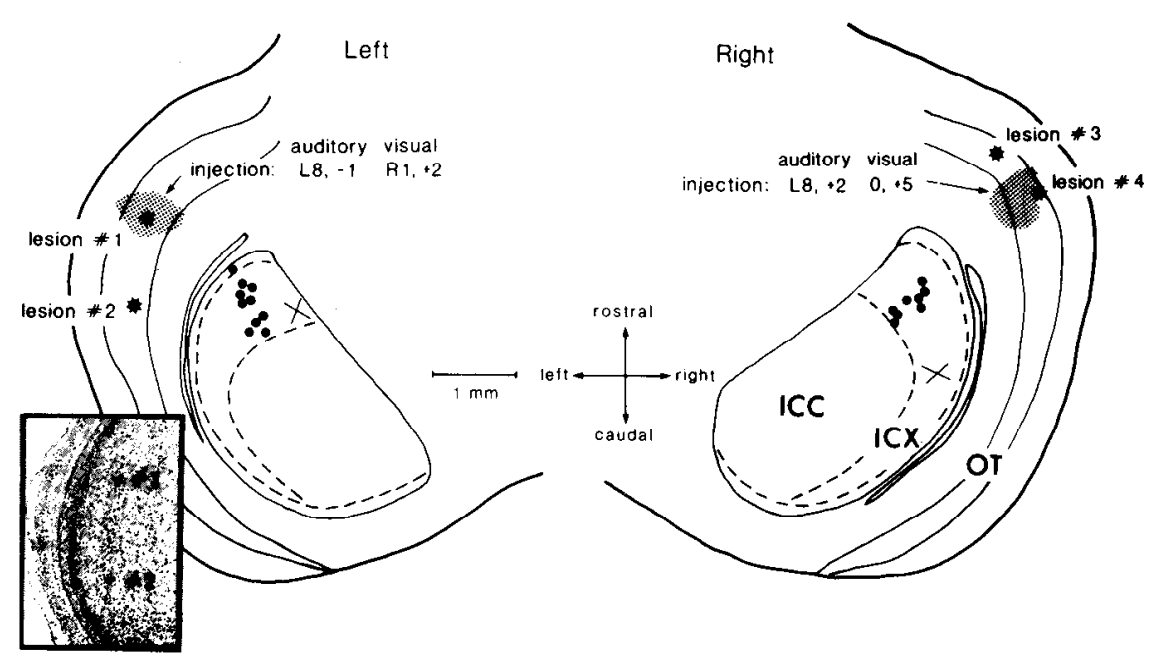

Owl \#4

data collected

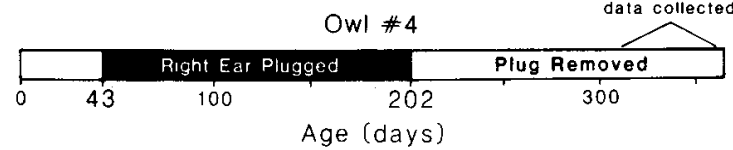


A

Plug In

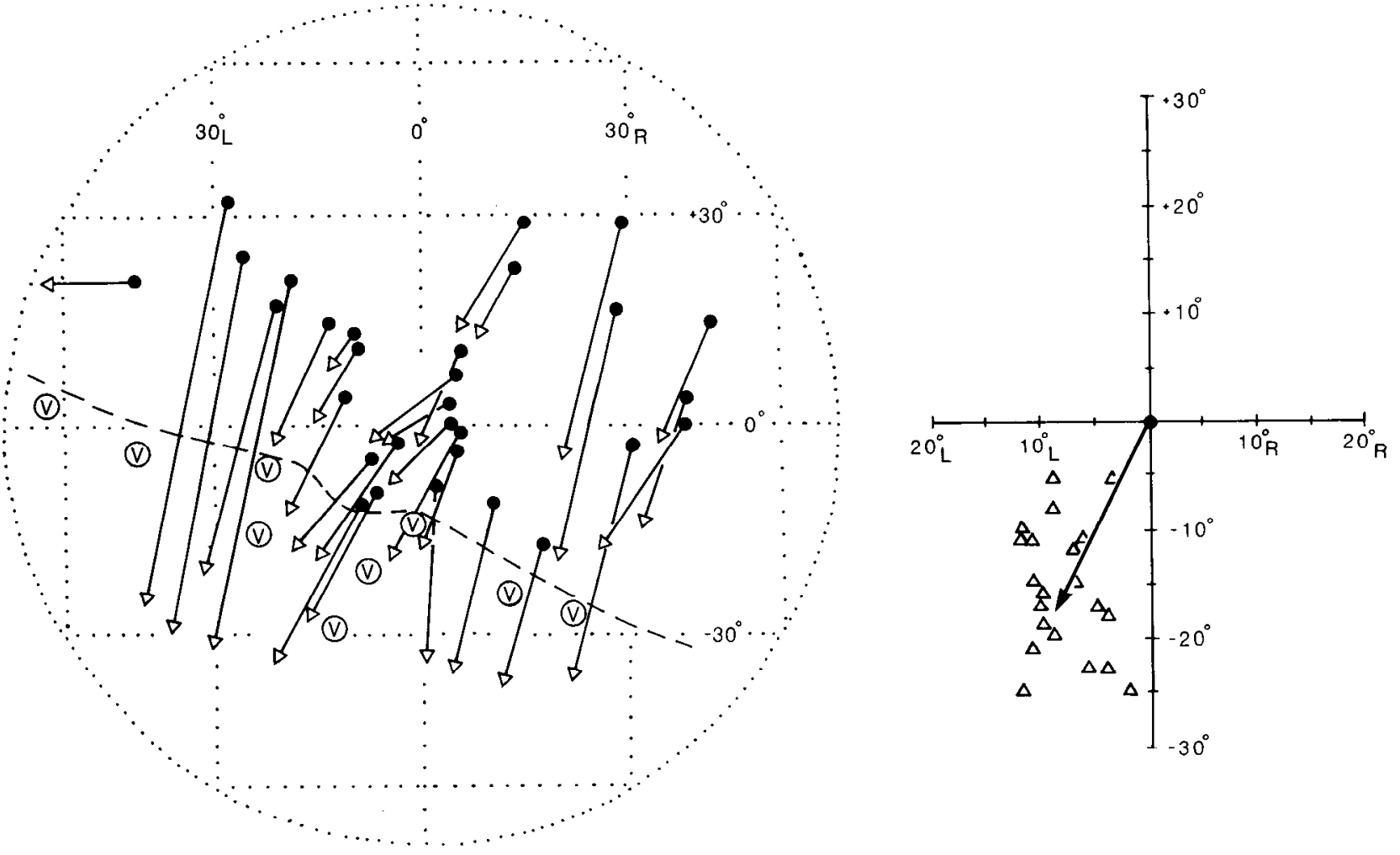

B Plug Removed
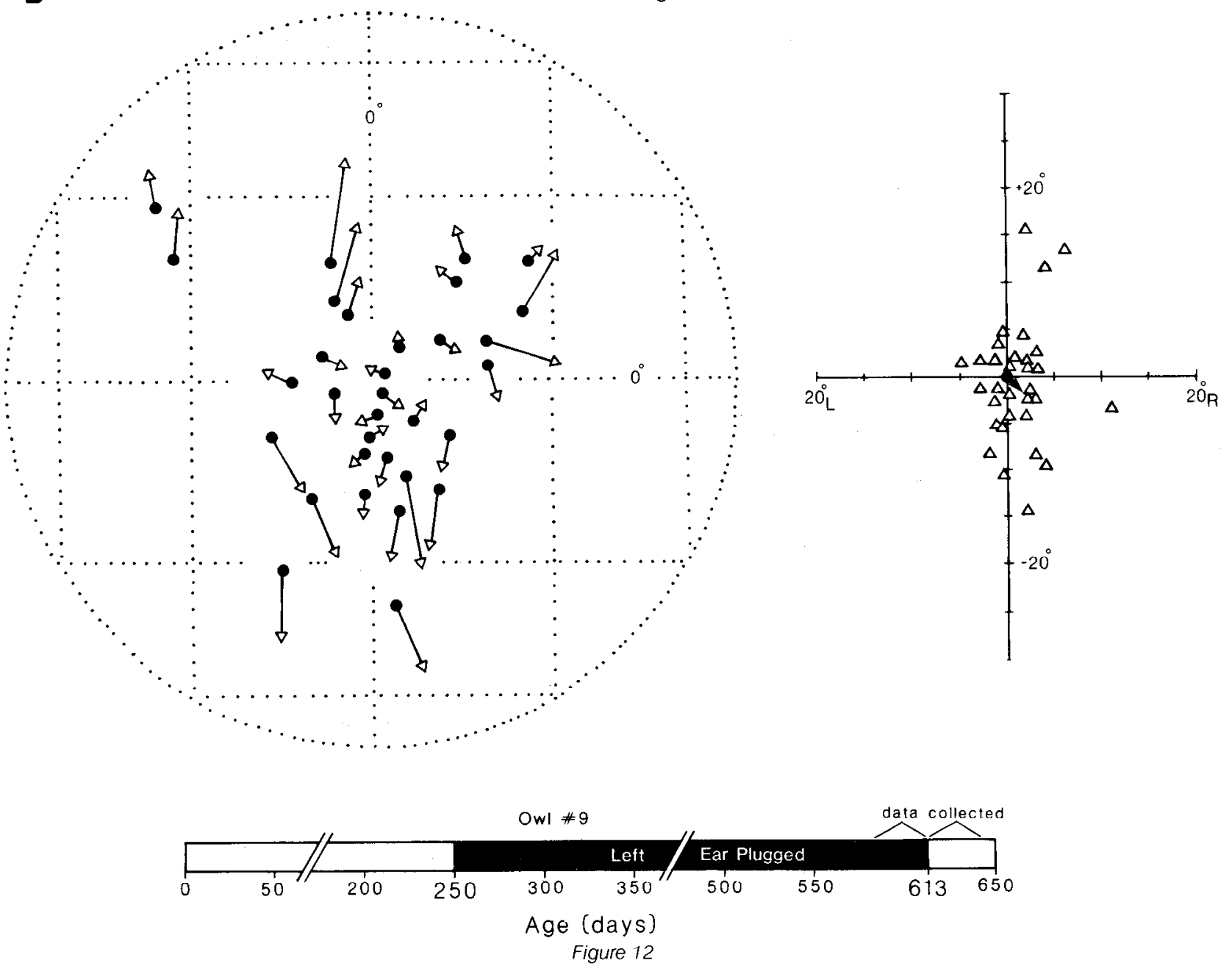
of owl 7 (Fig. 9). These data indicate that associations between auditory cues and locations in visual space become permanent or at least highly resistant to change, during a critical period in development. The end of this critical period is approximately coincident with the age at which barn owls reach sexual maturity.

Acute monaural occlusion in adult, control animals abolished auditory responses instead of causing a shift in auditory spatial tuning. This difference was probably due to the acute earplugs being more effective because they were fresh, whereas the chronic earplugs must certainly have deteriorated during the period of occlusion (see "Materials and Methods"). A previous study in normal owls has shown that acute monaural occlusion can either shift the spatial tuning of space-specific units or abolish their responses depending on the effectiveness of the earplug (Knudsen and Konishi, 1980).

Comparison of physiology with sound localization behavior. A comparison of the physiological data with the behavioral data collected from owis 4 and 5 reveals that audilory-visual misalignments measured physiologically in the optic tectum correspond roughly to sound localization errors measured behaviorally (Figs. 10 and 11). However, the variability in the behavioral responses was less than the variability in the alignment between auditory best areas and visual receptive fields, especially for owl 4 (Fig. 11). The magnitude and directions of auditory-visual misalignments varied across the tectum (for reasons that will be discussed later). The behavioral data do not reflect this variability because of the nature of the paradigm: the acoustic stimulus remained "on" until the owl settled on a particular direction. Thus, the owl's final head orientation was based on its judgment of a sound source which was located almost directly in front of its face. Therefore, the appropriate comparison is between the behavioral responses and the auditory-visual misalignments of units with receptive fields located directly in front of the bird. With this restriction, the match between the physiological and behavioral data is good (Fig. 11).

If the alignment of auditory best areas with visual receptive fields is a reliable correlate of sound localization, then the sensitive and critical periods that affect the development of sound localization (Knudsen et al., 1984a, b) should also affect the development of this alignment. This predicts that auditory spatial tuning can be modified by monaural occlusion only if it is imposed by 8 weeks of age (the end of the behavioral sensitive period) and that accurate auditory-visual alignment can be recovered following earplug removal only if normal auditory cues are restored by about 38 weeks of age (the proposed end of the behavioral critical period).

The data presented here are consistent with these predictions, with one minor exception: based on the data from owl 4, the physiological and behavioral critical periods end sooner than expected, at about 200 days ( 28 weeks) of age. The earplug was removed from owl 4 at 202 days of age. Five months later, this animal still had a substantial sound localization error and the alignment of auditory best areas and visual receptive fields in the tecta was equally inaccurate (Fig. 11). It is likely that ow 4 had even larger errors in sound localization and auditory-visual alignment immediately following earplug removal, and had adjusted partially during the intervening 4 months before measurements were taken. Partial adjustments of sound localization error were observed in three owls unplugged at between 200 and 270 days of age in a previous study (Knudsen et al.,. 1984b). Such partial adjustments may represent the capacity of the adult auditory system to make limited changes in the associations of auditory cues with locations in space after the critical period ends (Kriudsen, 1984c).

Effects of monaural occlusion on modality balance. Normally, tectal units responded strongly to both auditory and visual stimuli. However, in monaurally occluded birds with the earplug still in place or recently removed, most units (sometimes over $80 \%$ ) responded predominately or exclusively to one stimulus modality or the other (Fig. 3). Bimodal responsiveness returned to normal gradually over a period of weeks or months following the removal of the earplug.

This effect of monaural occlusion must be interpreted cautiously. The modality balance of a unit was classified on the basis of its responses to stimuli of each modality presented alone. Visual-only units (category 1) may well have responded to auditory stimuli, if both stimuli had been presented simultaneously, and the converse may have been true for auditory-only (category 7 ) units. Moreover, the proportional increase of visual-only units does not necessarily imply that auditory inputs were suppressed. The earplug may have created conditions under which the binaural requirements of units could never be met under free-field conditions (presumably, this was lhe case in owl 9 for units with low visual receptive fields; Fig. 12). Similarly, for units that altered their spatial tuning as a consequence of early monaural occlusion, removal of the earplug may have eliminated the binaural cues that they required, as was described for units in owl 5. However, this explanation does not account for the proportional increase in auditory-only units or the fact that, in owls 4 and 7, normal bimodal fractions were observed even though the auditory spatial tuning of units remained attered. Thus, it is possible that in young animals, chronic monaural occlusion, in addition to altering auditory spatial tuning, suppresses the degree of interaction between sensory modalities.

Effects of monaural occlusion on spatial selectivity. Monaural occlusion does not shift auditory space uniformly, the way a prism shifts visual space. Each cue can vary irregularly and in a complicated pattern as a function of sound source location, and an earplug alters differcmt auditory cues differently. This point is made by the differential effects of earplug insertion or removal on best areas at different locations (Figs. 9,11, and 12). The variability of the induced shift in elevation, which presumably reflects the sensitivity of units to interaural spectrum differences (Knudsen and Konishi, 1979; Knudsen, 1980; Moiseff and Konishi, 1981; 1983), was particularly large: in the same individual, best areas in one portion of space may move up while those in another portion may move down. However, the shifts were not random. They followed a definite pattern, probably representing the spatial pattern of interaural amplitude differences for that individual. The relatively small effect of monaural occlusion on best areas located in front of the animal implies that changes in interaural spectrum (frequency-specific differences in interaural amplitude) per degree of space are large in this region. Conversely, the large shifts in best areas located peripherally imply that, in these regions; interaural spectrum changes gradually with source location. The differential effect of earplugging on best areas at different locations almost certainly gives rise to the changes in the relative magnification of different portions of space observed previously (Knudsen, 1983a) and shown in Figures 9, 11, and 12.

The fact that monaural occlusion affects different portions of space differently also accounts for the observation that auditory receptive fields could contract or expand following insertion or removal of an earplug. Thus, a change in receptive field size does not necessarily imply that the selectivity of a unit for auditory cues has increased or decreased. The average size of auditory receptive fields remained fairly constant in occluded and non-occluded birds (Fig. 1), which argues that there was no systematic improvement or deterioration of unit selectivity for spatial cues:

"Neuronal deprivation." In all animals that were monaurally oc.

Figure 12. Alignment of auditory best areas with visual receptive fields in owl 9 recorded before $(A)$ and after $(B)$ earplug removal. Owl 9 was monaurally occluded as an adult. On the left, centers of best areas $(\Delta)$ and centers of visual receptive fields $(\mathcal{O})$ for individual units are connected by lines. The circled $V$ 's in $A$ indicate the visual receptive fields of units in the intermediate tectal layers that did not respond to auditory stimuli. All units with visual receptive fields located below the dashed line $(n=1 \dagger)$ could not be driven with acoustic stimuli, On the right, the centers of auditory best areas of all bimodal units located in the rostral tectum are plotted relative to the centers of their visual receptive fields. The vectors represent the median auditory-visual misalignments of these samples. The auditory history of owl 9 and the periods over which the data were collected are indicated at the bottom. 
cluded early in life, the responsiveness of units in the tectum was abnormal: they tended to discharge erratically or sluggishly and they often habituated to repeated stimulation. Sluggish and habituating responses have been reported in preparations in which animals have been deprived of sensory stimulation (Wiesel and Hubel, 1965; Hubel and Wiesel, 1970; Olson and Pettigrew, 1974; Stryker et al., 1978; Gordon et al., 1979). However, monaural occlusion does not deprive an animal of spatial cues, rather it alters the cues so that the correspondence between cues and locations is abnormal. Nevertheless, if tectal neurons were innately selective for a certain range of cue values, then these neurons would be "deprived" of adequate stimulation even as the animal experienced rich (but abnormal) localization cues. That the auditory system "expects" certain ranges of localization cues is supported by the ability of animals of various species, including humans, to localize sounds soon after birth (Wertheimer, 1961; Gottlieb, 1971; Clements and Kelly, 1978; Muir and Field, 1979; Olmstead and Villablanca, 1980) and by the fact that barn owls consolidate associations between "normal" cues and locations in space better than they consolidate associations based on abnormal cues (Knudsen et al., 1984b). Thus, the weakened responsiveness of tectal units in birds that were monaurally occluded before 8 weeks of age might represent the effects of "neuronal deprivation" resulting from their innate selectivity for limited ranges of binaural cues. Since an equivalent weakening of unit responsiveness was nol observed in owl 9, neurons must be susceptible to this "deprivation" effect only during an early period in development.

Properties of the adjustment process. Prolonged monaural occlusion, while altering the spatial tuning of units, had little effect on other auditory properties (Fig. 1). Apparently, the mechanism by which the spatial tuning of units is adjusted by experience does not involve changes in frequency tuning (CF or $Q$ ) or in degree of spatial selectivity (average receptive field width and height).

The process of adjustment seems gradual based on the progressive realignment of auditory best areas with visual receptive fields observed in owl 2 (Fig. 8). This suggests that the underlying mechanism changes the tuning of units for spatial cues along a continuum of cue values. This inference is consistent with the observation that, in the tecta of birds raised with one ear occluded and with the earplug still in place, the locations of best areas were biased in the direction of the car that was occluded (Figs. 5 and $7 \mathrm{~A}$ ). This bias would not be expected if "correct" localization cues were selected discretely, but would be expected if adjustment were a continuous process that followed a continuum and the error signal causing the adjustment contained a window of acceptable accuracy.

Previous studies on other species suggest that modulation of inhibition may play an important role in adjusting auditory spatial tuning. In cats, many space-selective units in the optic tectum are excited by input to one ear and inhibited by input to the other (Wise and Irvine, 1983). These are referred to as "El" units. Moore and Irvine (1981) demonstrated that, in cats raised with one ear canal ligated, the proportion of El units in the inferior colliculus was lower than in controls, and that this decrease was due to a decrease in the inhibitory influence of inputs coming from the untouched ear. This effect is appropriate to counteract the effects of sound attenuation at the ligated ear on the spatial tuning of these units. Silverman and Clopton (1977) also reported changes in inhibitory effects on units in the inferior colliculus of mice. However, in their preparation inhibition from the ligated ear decreased (opposite to the effect described in the cat). Since inhibitory influences play a crucial role in establishing the spatial tuning of tectal units in the barn owl (Knudsen, 1984a), experience-dependent alterations in these influences might underlie the shifts in best areas (and the appearance of unlimited receptive fields).

The site of plasticity. Ultimately, we would like to determine where in the brain these experience-dependent changes take place. A point-to-point projection from the external nucleus of the ICX to the optic tectum brings the auditory space map, which is synthesized in the $\mathrm{ICX}$, to the tectum where it converges wilh the visual map of space (Knudsen and Knudsen, 1983; Knudsen, 1983b; 1984b). It was possible that the realignment of auditory best areas with visual receptive fields that was observed in the optic tectum was due to a remapping of the topographic projection from the ICX to the tectum, in a manner analogous to the remapping of visual projections to the tectum that has been demonstrated in other preparations (Keating, 1975; Schmidt et al., 1978; Meyer, 1979; Udin and Keating, 1981). The anatomical data from owl 4 domonstrate that this is not the case (Fig. 11C). The auditory spatial tuning of units in this bird had been shifted by $10^{\circ}$ in azimuth. Because frontal space is magnified greatly in the ICX space map (Knudsen and Konishi, 1978; Knudsen and Knudsen, 1983), a change in the topography of the ICX-totectum projections corresponding to a $10^{\circ}$ shift in azimuth would have been quite apparent ( $X$ 's in Fig. 11C). No hint of such an altered projection could be seen on either side. Another possibility is that the auditory realignment was due to alterations in connections intrinsic to the tectum. This hypothesis predicts a population of shortlatency units with unaltered auditory spatial tuning. Such a population would have been conspicuous, with dramatically different spatial tuning from neighboring units, and was not observed. Therefore, the data indicate that the experience-dependent changes must have occurred at or before the ICX.

It is logical for the experience-dependent alterations to occur at an early stage in the auditory pathway. Earplugs attenuate and delay different frequencies of sound differently (see "Materials and Methods"). The frequency tuning of tectal units is broad and essentially unchanged by ear occlusion (Fig. 1), whereas spatial tuning is sharp, but shifted. This implies that the spatial cues provided by each frequency band are reinterpreted independently, which must take place before the information from different frequency bands is inlegrated in the ICX (Knudsen, 1984b).

The experience-dependent changes may occur at the initial sites of binaural interaction, such as in nucleus laminaris and in the posterior division of the nucleus of the lateral lemniscus, where the tuning for interaural differences is broad and frequency tuning is sharp (Moiseff and Knoishi, 1983). Changes at this level might be accomplished by modulations in synaptic efficacy or changes in dendritic morphology as well as by the formation of new connections. In addition, if adjustments were made at this early level, then all subsequent processing of spatial cues could be carried out normally, i.e., the spatial information would be correct from the outset.

\section{References}

Clements, M., and J. B. Kelly (1978) Auditory spatial responses of young guinea pigs (Cacia porcellus) during and after ear blocking. J. Comp. Physiol. Psychol. 92: 34-44

Daw, N. W., and H. J. Wyatt (1976) Kittens reared in undirectional environment: Evidence for a critical period. J. Physiol. (Lond.) 257: 155-170.

Gordon, B., J. Presson, J. Packwood, and R. Schcer (1979) Alteration of cortical orientation selectivity: Importance of asymmetric input. Science 204: 1109-1111

Gottlieb, G. (1971) Development of Species Identification in Birds, University of Chicago Press, Chicago.

Hubel, D. H., and T. N. Wiesel (1970) The period of susceptibility to the physiological ettects of unilateral eye closure in kittens. J. Physiol. (Lond.) 206: $419-436$

Keating, M. J. (1975) The time course of experience-dependent synaptic switching of visual connections in Xenopus laevis. Proc. R. Soc. Lond. (Biol.) 198: 603-610

Knudsen, E. I. (1980) Sound localization in birds. In Comparative Studies of Hearing in Vertebrates, A. N. Popper and R. R. Fay, eds., pp. 287-322, Springer-Verlag, New York.

Knudsen, E. I. (1982) Auditory and visual maps of space in the optic tectum of the owl. J. Neurosci. 2: 1177-1194.

Knudsen, E. I. (1983a) Early auditory experience aligns the auditory map of space in the optic tectum of the barn owl. Science 222: 939-942.

Knudsen, E. I. (1983b) Subdivisions of the inferior colliculus in the barn owl (Tyto alba). J. Comp. Neurol. 218: 174-186.

Knudsen, E. I. (1984a) Auditory properties of space-tuned units in owl's optic tectum. J. Neurophysiol. 52: 709-723. 
Knudsen, E. I. (1984b) Synthesis of a neural map of auditory space in the owl. In Dynamic Aspects of Neocortical Functions, G. M. Edelman, W. M. Cowan, and W. E. Gall, eds., pp. 375-396, John Wiley \& Sons, Inc., New York.

Knudsen, E. I. (1984c) The role of auditory experience in the development and maintenance of sound localization. Trends Neurosci. 7: 326-330.

Knudsen, E. I., and P. F. Knudsen (1983) Space-mapped auditory projections from the inferior colliculus to the optic tectum in the barn owl (Tyto alba). J. Comp. Neurol. 218: 187-196.

Knudsen, E. I., and M. Konishi (1978) A neural map of auditory space in the owl. Science 200: 795-797

Knudsen, E. I., and M. Konishi (1979) Mechanisms of sound localization by the barn owl (Tyto alba). J. Comp. Physiol. 133: 13-21.

Knudsen, E. I., and M. Konishi (1980) Monaural occlusion shifts receptivefield locations of auditory midbrain units in the owl. J. Neurophysiol. 44: 687-695

Knudsen, E. I., G. G. Blasdel, and M. Konishi (1979) Sound localization by the barn owl measured with the search coil technique. J. Comp. Physiol. 133: $1-11$

Knudsen, E. I., P. F. Knudsen, and S. D. Esterly (1982) Early auditory experience modifies sound localization in barn owls. Nature 295: 238240.

Knudsen, E. I., S. D. Esterly, and P. F. Knudsen (1984a) Monaural occlusion alters sound localization during a sensitive period in the barn owl. J. NeurosCl. 4: 1001-1011.

Knudsen, E. I., P. F. Knudsen, and S. D. Esterly (1984b) A critical period for the recovery of sound localization accuracy following monaural occlusion in the barn owl. J. Neurosci. 4: 1012-1020.

Leibovic, S. J., and E. I. Knudsen (1982) Recovery of spatial tuning of auditory neurons in the owl's optic tectum. J. Acoust. Soc. Am. Suppl. 72: 541

Meyer, R. L. (1979) Retinotectal projection in goldfish to an inappropriate region with a reversal in polarity. Science: $205: 819-821$.
Moiseff, A., and M. Konishi (1981) Neuronal and behavioral sensitivity to binaural time differences in the owl. J. Neurosci. 1: 40-48.

Moiseff, A., and M. Konishi (1983) Binaural characteristics of units in the owl's brainstem auditory pathway: Precursors of restricted spatial receptive fields. J. Neurosci. 3: 2553-2562.

Moore, D. R., and D. R. Irvine (1981) Plasticity of binaural interaction in the cat inferior colliculus. Brain Res. 208: 198-202.

Muir, D., and J. Field (1979) Newborn infants orient to sounds. Child Dev. 50: $431-436$

Olmstead, C. E., and J. R. Villablanca (1980) Development of behavioral audition in the kitten. Physiol. Behav. 24: 705-712.

Olson, C. R., and J. D. Pettigrew (1974) Single units in visual cortex of kittens reared in stroboscopic illumination. Brain Res. 70: 189-204.

Schmidt, J. T., C. M. Cicerone, and S. S. Easter (1978) Expansion of the half retinal projection to the tectum in goldfish. An electrophysiological and anatomical study. J. Comp. Neurol. 177: 257-278.

Silverman, M. S., and B. M. Clopton (1977) Plasticity of binaural interaction. I. Effect of early auditory deprivation. J. Neurophysiol. 40: 1266-1274.

Stryker, M. P., H. Sherk, A. G. Leventhal, and H. V. B. Hirsch (1978) Physiological consequences for the cat's visual cortex of effectively restricting early visual experience with oriented contours. J. Neurophysiol. 41: 896-909.

Udin, S. B., and M. J. Keating (1981) Plasticity in a central nervous pathway in Xenopus: Anatomical changes in the isthmotectal projection after larval eye rotation. J. Comp. Neurol. 203: b/b-594.

Wertheimer, M. (1961) Psychomotor coordination of auditory and visual space at birth. Science 134: 1692

Wiesel, T. N., and D. H. Hubel (1965) Comparison of the effects of unilateral and bilateral eye closure on cortical unit responses in kittens. J. Neurophysiol. 28: 1029-1040.

Wise, L. Z., and D. R. F. Irvine (1983) Auditory response properties of neurons in deep layers of cat superior colliculus. J. Neurophysiol. 49:674685. 